\title{
Characteristics of north jovian aurora from STIS FUV spectral images
}

\author{
J. Gustin ${ }^{\text {a,* }}$, D. Grodent ${ }^{\text {a }}$, L.C. Ray ${ }^{\text {b }}$, B. Bonfond ${ }^{\text {a }}$, E.J. Bunce ${ }^{c}$, J.D. Nichols ${ }^{c}$, N. Ozak ${ }^{\mathrm{d}}$ \\ ${ }^{a}$ Laboratoire de Physique Atmosphérique et Planétaire, Université de Liège, B-4000 Liège, Belgium \\ ${ }^{\mathrm{b}}$ Department of Physics and Astronomy, University College London, London, UK \\ ${ }^{c}$ Department of Physics and Astronomy, University of Leicester, Leicester LE1 7RH, UK \\ ${ }^{\mathrm{d}}$ Department of Earth and Planetary Sciences, Weizmann Institute of Science, 76100 Rehovot, Israel
}

\section{A R T I C L E I N F O}

\section{Article history:}

Received 17 July 2015

Revised 20 October 2015

Accepted 19 December 2015

Available online 6 January 2016

\section{Keywords:}

Aurorae

Hubble Space Telescope observations

Jupiter, atmosphere

Jupiter, magnetosphere

Spectroscopy

\begin{abstract}
A B S T R A C T
We analyzed two observations obtained in Jan. 2013, consisting of spatial scans of the jovian north ultraviolet aurora with the HST Space Telescope Imaging Spectrograph (STIS) in the spectroscopic mode. The color ratio (CR) method, which relates the wavelength-dependent absorption of the FUV spectra to the mean energy of the precipitating electrons, allowed us to determine important characteristics of the entire auroral region. The results show that the spatial distribution of the precipitating electron energy is far from uniform. The morning main emission arc is associated with mean energies of around $265 \mathrm{keV}$, the afternoon main emission (kink region) has energies near $105 \mathrm{keV}$, while the 'flare' emissions poleward of the main oval are characterized by electrons in the 50-85 keV range. A small scale structure observed in the discontinuity region is related to electrons of $232 \mathrm{keV}$ and the Ganymede footprint shows energies of $157 \mathrm{keV}$. Interestingly, each specific region shows very similar behavior for the two separate observations.

The Io footprint shows a weak but undeniable hydrocarbon absorption, which is not consistent with altitudes of the Io emission profiles $(\sim 900 \mathrm{~km}$ relative to the 1 bar level) determined from HST-ACS observations. An upward shift of the hydrocarbon homopause of at least $100 \mathrm{~km}$ is required to reconcile the high altitude of the emission and hydrocarbon absorption.

The relationship between the energy fluxes and the electron energies has been compared to curves obtained from Knight's theory of field-aligned currents. Assuming a fixed electron temperature of $2.5 \mathrm{keV}$, an electron source population density of $\sim 800 \mathrm{~m}^{-3}$ and $\sim 2400 \mathrm{~m}^{-3}$ is obtained for the morning main emission and kink regions, respectively. Magnetospheric electron densities are lowered for the morning main emission $\left(\sim 600 \mathrm{~m}^{-3}\right)$ if the relativistic version of Knight's theory is applied.

Lyman and Werner $\mathrm{H}_{2}$ emission profiles, resulting from secondary electrons produced by precipitation of heavy ions in the 1-2 MeV/u range, have been applied to our model. The low CR obtained from this emission suggests that heavy ions, presumably the main source of the X-ray aurora, do not significantly contribute to typical UV high latitude emission.
\end{abstract}

(c) 2016 Elsevier Inc. All rights reserved.

\section{Introduction}

\subsection{Background}

The ultraviolet jovian aurora is mainly produced by the interaction between the $\mathrm{H}_{2}$ atmosphere and precipitating magnetospheric electrons. In the far ultraviolet (FUV, between 1200 and $1700 \AA$ ), the emission is dominated by the Lyman- $\alpha$ line from atomic hydrogen resulting from $\mathrm{H}_{2}$ dissociation and $\mathrm{H}_{2}$ vibronic lines from the Lyman $\left(B^{1} \Sigma_{g}^{+} \rightarrow X^{1} \Sigma_{g}^{+}\right)$and Werner $\left(C^{1} \prod_{u}^{+} \rightarrow X^{1} \Sigma_{g}^{+}\right)$system bands. The auroral emission is known to interact with the atmosphere

\footnotetext{
* Corresponding author.
}

through absorption by the main hydrocarbons. Methane $\left(\mathrm{CH}_{4}\right)$ attenuates the emission at wavelengths below $1400 \AA$, ethane $\left(\mathrm{C}_{2} \mathrm{H}_{6}\right)$, which has a continuous absorption cross-section shortward of $1550 \AA$, has a typical signature between 1400 and $1480 \AA$ in the case of strongly attenuated spectra, and acetylene $\left(\mathrm{C}_{2} \mathrm{H}_{2}\right)$ which has a more peaked cross-section and has a significant effect at $1480 \AA$ and $1520 \AA$. This very wavelength-dependent absorption is measured by the color ratio $C R=I(1550-1620 \AA) / I(1230$ $1300 \AA$ ) with $I$ the brightness in photons unit, originally introduced in a slightly different form by Yung et al. (1982). The CR is directly correlated with the amount of hydrocarbon at or above the $\mathrm{H}_{2}$ auroral emission and is related to the altitude of the aurora relative to the hydrocarbons (Gustin et al., 2013b). It is thus an important tool to determine the energy of the primary electrons precipitating 
into the atmosphere. While broadband images are commonly used to characterize spatial and temporal effects of the aurora, FUV spectra allow the determination of the mean energy $\langle E\rangle$ of the precipitating electrons and improve our knowledge of the processes leading to the different auroral regions.

UV images obtained with Hubble Space Telescope (HST) cameras (STIS in the imaging mode and the Advanced Camera for Survey (ACS)) have shown a complex and dynamical auroral structure and revealed that the auroral morphology is characterized by several distinct components of emission.

The first component is the main oval, which maps magnetically to the magnetosphere between $\sim 20$ and $\sim 30$ jovian radius $\left(R_{\mathrm{J}}\right)$ in the equatorial plane, and results from the magnetosphere-iono sphere coupling current system associated with the breakdown of corotation in the middle magnetosphere, specifically with the region of upward field-aligned currents (e.g. Cowley and Bunce, 2001). This emission has an apparent brightness generally in the $35-450 \mathrm{kR}$ range of $\mathrm{H}_{2}$ in the Lyman and Werner bands ( $1 \mathrm{kR}=10^{9}$ photons per $\mathrm{cm}^{2}$ per sec emitted in $4 \pi$ steradians). Occasionally, emissions of a few MR have been observed in the morning sector. The main emission is characterized by a narrow bright arc extending from dawn to noon or further. This main emission generally becomes more diffuse and weaker in the afternoon sector than in the morning sector, with a 'kink' region in the $110-150^{\circ}$ System III $\left(\mathrm{S}_{\text {III }}\right)$ sector of the northern aurora, associated with a localized magnetic anomaly (Grodent et al., 2008). Timetagged observations show that the main oval energy flux usually varies steadily over several minute intervals and is somewhat correlated with the mean energy of the precipitating electrons, such that the current density (i.e. the electron flux) remains relatively constant (Gustin et al., 2004a). Assuming an emission exclusively due to electrons, the auroral mean electron energy lies within the range $35-200 \mathrm{keV}$. Two cases with harder electrons ( $\sim 280 \mathrm{keV}$ and $\sim 460 \mathrm{keV}$ ) have also been revealed from a STIS observation of an exceptionally bright morning aurora (Gustin et al., 2006). Overall, Gustin et al. (2004a) showed that the main oval properties are consistent with Knight's theory of auroral electron acceleration associated with field-aligned current flow (Knight, 1973), involving a magnetospheric source of electrons with a thermal energy of $2.5 \mathrm{keV}$ and with a density varying between 1000 and $10,000 \mathrm{~m}^{-3}$, consistent with "warm" magnetospheric electrons observed by Voyager (Scudder et al., 1981). A discontinuity region in the main emission, characterized by a significant drop of the brightness and fixed between 08:00 and 13:00 magnetic local time (MLT) has also been identified in HST images (Radioti et al., 2008). Work by Chané et al. (2013) suggests that this discontinuity is the result of an asymmetry in the thermal pressure distribution, decreasing the strength of the corotation current system in that sector. Finally, a transient small-scale structure in the main emission, close to magnetic noon, has recently been identified by Palmaerts et al. (2014). This structure is present in both hemispheres, located between 09:00 and 15:30 MLT in the northern hemisphere and between 10:00 and 15:00 MLT in the southern hemisphere. Intermittent plasma flow in the equatorial plane near the noon sector is advanced as a possible explanation for this emission.

The second component, located poleward of the main oval, consists of scattered diffuse emissions, generally weaker than the main oval ( $\sim 15$ to $\sim 70 \mathrm{kR})$. These "polar cap" emissions exhibit large time variations on timescales of minutes or less ("flares"). Occasionally, very dynamical, intense and sometimes quasi-periodic brightenings of several MR have been observed, possibly related to abrupt solar wind variations (Waite et al., 2001; Bonfond et al., 2011). The latter bright polar flares have never been directly observed with UV spectrographs, which makes it problematic to determine their characteristics, but STIS spectra obtained from "typical" polar emissions are generally associated with mean electron energies in the $30-100 \mathrm{keV}$ range. Values up to $\sim 180 \mathrm{keV}$ have been observed but are exceptional, considering the few observations available. Furthermore, the energy fluxes and current densities associated with polar emissions determined by Gustin et al. (2004a) are significantly lower than main oval values ( $\leqslant 10 \mathrm{~mW} \mathrm{~m}^{-2}$ and $\leqslant 0.12 \mu \mathrm{A} \mathrm{m}^{-2}$ for polar regions compared to values up to $30 \mathrm{~mW} \mathrm{~m}^{-2}$ and $0.3 \mu \mathrm{A} \mathrm{m}^{-2}$ for the energy flux and current density, respectively, of the main oval). At the higher end of the energy flux range in this region, the auroral acceleration mechanism may be similar to that in the main oval, while the weaker emissions are not clearly associated with field-aligned currents and could result from precipitation from a quasi-isotropic hot magnetospheric electron source (Gustin et al., 2006). The time evolution of $\langle E\rangle$ generally shows little correlation (positive or negative) with the energy flux precipitated during these transient events. This behavior suggests that the mechanism responsible for rapid transient brightenings does not increase the energy of the precipitated electrons, but it enhances their number flux (Gérard et al., 2003).

The third type of auroral emission consists of spots at the magnetic footprints of Io, Ganymede and Europa, observed both in UV and in infrared wavelengths (Prangé et al., 1996, 1998; Grodent et al., 2006; Clarke et al., 1996, 1998, 2002). The Io footprint (IFP) consists of several spots observed in both hemispheres (Bonfond et al., 2009), followed by a trailing tail that sometimes extends for more than $180^{\circ}$ in longitude (Clarke et al., 1998; Gérard et al., 2002). The IFP brightness in the Lyman and Werner $\mathrm{H}_{2}$ bands may be very variable with time and location, as spectra and images of the IFP shows observed values from $35 \mathrm{kR}$ (e.g. Bonfond et al., 2009), with peaks of up to $670 \mathrm{kR}$ (e.g. Gérard et al., 2002) and all intermediate values (Wannawichian et al., 2010). The vertical brightness of the IFP spots can go up to 6 MR and depend on both the $S_{\text {III }}$ longitude of the satellite and the hemisphere considered (Bonfond et al., 2013). The IFP spectral observations are characterized by remarkably stable CR from 1.4 to 2.3. Compared to the other auroral regions, where the CR can vary from 1.4 to more than 10 , these IFP color ratios (CRs) correspond to precipitating electrons of mean energy between $40 \mathrm{keV}$ and $70 \mathrm{keV}$ (Gérard et al., 2002). These values were obtained from the methane vertical profile and the pressure-altitude relationship in the North Equatorial Belt (NEB) atmosphere from Gladstone et al. (1996), adapted to gravity at the polar region. Study of limb profiles by Bonfond et al. (2009) shows that the peak of IFP emission profile is situated between 550 and $1300 \mathrm{~km}$. The peak altitude, at $\sim 900 \mathrm{~km}$ on average, is found to be relatively constant with distance from the main spot. By using Monte Carlo simulations, these authors showed that an observed emission peaking at $900 \mathrm{~km}$ is compatible with precipitation of electrons distributed as a kappa function with a mean energy of $\sim 1.1 \mathrm{keV}$, much lower than the values obtained by Gérard et al. (2002). This discrepancy, still not elucidated, is usually interpreted as being the result of our poor knowledge of the hydrocarbon density profiles in polar regions, which is thought to be very different from observed and modeled profiles determined at low latitudes because of the energy deposition induced by the aurora.

A fourth component consist of emissions equatorward of the main emission: transient emissions possibly due to magnetospheric injections (Dumont et al., 2014) and diffuse emissions, presumably associated with electron scattering by whistler mode waves (Radioti et al., 2009).

\subsection{Objectives of the study}

Contrary to the auroral brightness, easily monitored by the thousands of images obtained with HST instruments, the factors 
which influence the primary electron mean energy variations are difficult to interpret for two reasons. First, the limited number of spectral observations available has been obtained through the long 1997-2009 period and thus reflects various solar and magne tospheric-ionospheric conditions, which make it difficult to directly compare different measurements. Second, the observed spectra either cover a limited portion of the aurora due to the small size of the aperture projected onto the planet (e.g. Gladstone and Skinner, 1989 with the International Ultraviolet Explorer, Dols et al. (2000) with the Goddard High resolution spectrograph onboard HST, Gustin et al. (2002, 2004a, 2006) and Gérard et al. (2002, 2003) with the HST/STIS in the spectroscopic mode), or integrated the whole polar region without spatial resolution (e.g. observations with the Far Ultraviolet Spectroscopic Explorer by Gustin et al., 2004b). Such former spectroscopic observations thus do not allow us to draw a global picture of the processes responsible for polar auroras.

Spectral images of the jovian aurora obtained recently with STIS allow a quasi-instantaneous diagnostic of the energy of the precipitating electrons. First, CR maps derived from the two spectral observations of the Northern pole obtained on 16 and 24 Jan. 2013 through the G012883 HST program revealed that the spatial distribution of the mean energy is far from uniform (Gustin et al., 2013a, 2014). Several regions show little absorption (e.g. the Io footprint or the discontinuity region), while others are significantly attenuated (e.g. the main oval morning arc and flare emissions). A confined emission situated near the noon sector, also shows strong hydrocarbon attenuation. This emission, associated with the Ganymede footprint in both observations presented by Gustin et al. (2013a, 2014) is now identified as the small scale structure described by Palmaerts et al. (2014) in the 16 Jan. 2013 observation. Four more STIS spectral images obtained in 2014 (three of the Northern and one of the Southern hemisphere) also revealed such spatial inhomogeneity of the energy distribution (Gérard et al., 2014). These authors used mono-energetic precipitating electrons to derive the mean energy of the auroral electrons. Depending on the region probed, the electron energy varies from a few keV up to $700 \mathrm{keV}$.

The two sets of observations used in the present study consist of a continuous time-tagged spatial scan of the north jovian polar region with the STIS $52^{\prime \prime} \times 0.5^{\prime \prime}$ slit in spectroscopic mode. This procedure allows us to obtain reconstructed images of the aurora, where each pixel contains spectral information and makes it possible to derive the CR and estimate the energy of the precipitating electrons for each pixel. The present data thus exhibit a quasisimultaneous picture of the whole northern aurora, from which several regions may be extracted and compared. In particular, this dataset allows for the first time the analysis of the 'small scale structure' and the Ganymede footprint auroral emissions. The results described in this paper will bring new insight on auroral characteristics and will be discussed within the frame of the current models describing the mechanisms responsible for the auroral phenomenon at Jupiter. Section 2 presents the dataset. In Section 3 and Appendix A, we describe the model that links the distribution of electrons precipitating at the top of the atmosphere to the CR. In Section 4, several representative zones of the aurora are inspected, from which the energy-flux relationship and the energy and brightness distributions are examined. In addition to the common interpretation that assumes that UV aurora is primarily created by electrons, the hypothesis of UV high latitude emission created by precipitation of heavy ions has been explored. Section 5 summarizes our results and provides the conclusions of this study.

\section{Observations and data reduction}

This study is based on two STIS observations obtained on 16 Jan. and 24 Jan., 2013, operated in the long slit spectral mode, as part of the G012883 HST program. The G140L grating was used along with the $52^{\prime \prime} \times 0.5^{\prime \prime}$ aperture and the FUV-MAMA detector, providing spatially resolved spectra in the range 1150-1750 $\AA$ with $\sim 12 \AA$ spectral resolution. For MAMA first-order modes, only $25^{\prime \prime}$ of the slit's length projects on the detector. The spectral range includes both absorbed and unabsorbed $\mathrm{FUV} \mathrm{H}_{2}$ emission, which is ideal to derive color ratios. The HST performed a continuous slew to move the slit projection from above the north limb to the equatorward edge of the aurora. The observations were designed to optimize the angle between the slit and the planetary equator in order to maximize the chances of intercepting the whole aurora during the scan. The two observations have an exposure time of $2528.2 \mathrm{~s}$ and were obtained in the time-tag mode, where the position and detection time of every photon is recorded in an event list with a $125 \mu$ s precision. In order to correctly identify each auroral feature and compute the $\chi$ angle (angle between the local normal and the observer) for each point, we reconstructed orthonormed images corresponding to chosen wavelength ranges based on the time-tagged event list. The orientation of the slit relative to the sky remained constant during each exposure. However, the motion of the center of slit relative to Jupiter involves a translation along a rotating axis as well as a compensation for the motion of Jupiter relative to the sky. In order to recompose orthonormed images with the $Y$-axis corresponding to the slit axis, it is necessary (1) to adjust the integration time corresponding to each row so that the angle covered by the motion of slit perpendicular to it equals the STIS platescale (i.e. $0.024^{\prime \prime}$ ) and (2) shift this row to account for the motion of the center of the slit along an axis parallel to it. To do so, the motion of the slit relative to the sky is retrieved from the observational log file (the "jitter" file) and the motion of Jupiter is derived from the ephemeris. For each time step, a calibrated 2D spectrum is built out of the time-tagged event list using the standard CALSTIS pipeline. Then the brightness is integrated over the appropriate spectral window in order to produce a row on the orthonormed image. Once such image is built, we manually localize the $X$ and $Y$ position of IFP main spot. Since each row of the image corresponds to a different time, we compute the $S_{\text {III }}$ longitude of Io corresponding to this time and then the longitude and latitude of the IFP main spot, using the reference oval from Bonfond et al. (2009). Based on this information, it is then possible to infer the $X$ and $Y$ location of the center of Jupiter on the image. Such a location is crucial to build the polar projection map corresponding to each observation, as it allows us to provide a latitude and a $\mathrm{S}_{\text {III }}$ longitude for each point on the image, acknowledging that the CML is different for each row since it varies with time. Unlike a true image where each pixel is simultaneously illuminated by the source, images reconstructed by this method should be called "pseudo-images", as 42 min were necessary to perform the scan of the North pole. Fig. 1 shows maps of the unabsorbed $\mathrm{H}_{2}$ brightness of the aurora in the whole UV bandwidth (700$1700 \AA$ ) for the 16 Jan. 2013 observation (obs. \#1, Fig. 1a and b) and 24 Jan. 2013 (obs. \#2, Fig. 1c and d). These images were obtained by integrating the spectrum corresponding to each spatial pixel in the 1550-1620 Å bandwidth, unaffected by hydrocarbon absorption, and multiplied by 8.1 to extend the result over the whole UV. The latter factor has been determined from a synthetic $\mathrm{H}_{2}$ spectrum as described by Gustin et al. (2004b). All brightnesses mentioned hereafter correspond to unabsorbed emission for the whole UV bandwidth. The two observations display very comparable features, which correspond to the different regions of the 

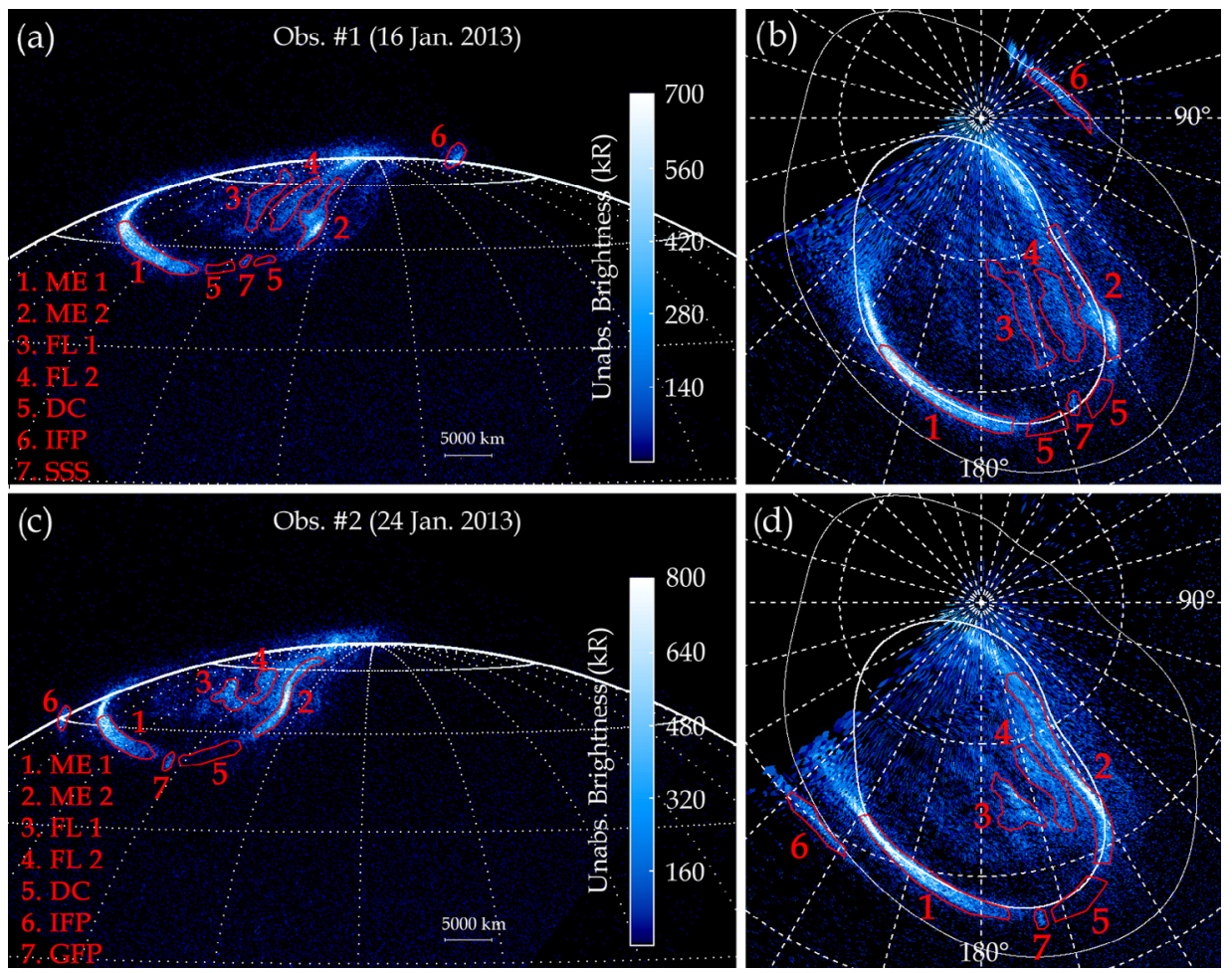

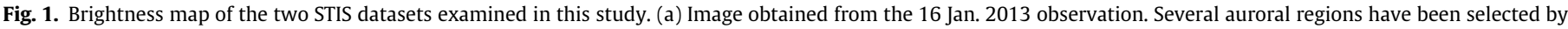

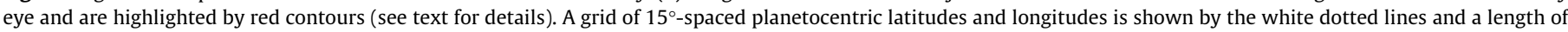

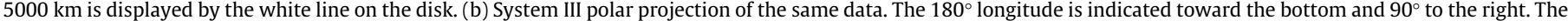

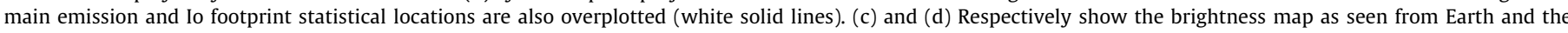

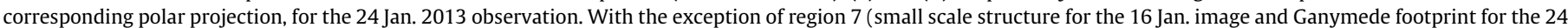

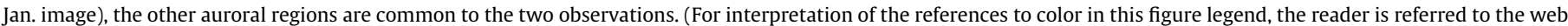
version of this article.)

aurora described in Section 1. These regions were manually selected from the images (red contours in Fig. 1) and are discussed in details throughout the paper. First, a very bright main arc in the morning sector (ME 1), with a distribution of emission in the $50-600 \mathrm{kR}$ range (centered at $\sim 275 \mathrm{kR}$ ), evolving to a discontinuity between $150^{\circ}$ and $185^{\circ} \mathrm{S}_{\text {III }}$ longitude, characterized by a very faint emission (mostly around $25 \mathrm{kR}$ and $<60 \mathrm{kR}$ ). As the morning arc, the main emission in the afternoon sector (ME 2) is also very bright (in the $25-800 \mathrm{kR}$ range centered around $240 \mathrm{kR}$ ), but is more spread out. Emissions inside the main oval are also present (FL 1, FL 2), taking the form of diffuse emissions, mostly in the range 30-300 kR. The Io footprint (IFP), clearly visible in both images, is characterized by pixels of brightness between 40 and $300 \mathrm{kR}$, centered at $\sim 165 \mathrm{kR}$. The small scale structure (SSS) is present on obs. \#1. It is situated within the discontinuity region (thus unaffected by the bright main emission) and exhibits pixels with very scattered brightnesses, between $\sim 20$ and $\sim 170 \mathrm{kR}$. The Ganymede footprint is visible on obs. \#2 and also shows a wide range of brightness values, from 40 to $240 \mathrm{kR}$. It should be stressed that these brightnesses correspond to observed values and are thus influenced by viewing angle effects. Signal from the pixels corresponding to these different regions has been extracted from the images as highlighted in Fig. 1, and are discussed in detail in the following sections.

\section{Mean energy of the primary electrons}

\subsection{Overview of the auroral model}

The ratio between the images integrated in the 1550-1620 and the $1230-1300 \AA$ ranges directly provides a map of the CR, which in turn can be converted to mean energy $\langle E\rangle$ of the precipitating electrons. The necessary prerequisite to build a map of $\langle E\rangle$ is to set up a model that relates CR to $\langle E\rangle$ and accounts for the emission angle $\chi$. Indeed, at first order, the thickness of the hydrocarbon layer crossed by the auroral photons varies with $\cos (\chi)$ and influences the observed CR. The emission angle thus affects the amount of hydrocarbon absorption perceived by the observer, and hence the determination of $\langle E\rangle$.

Another aspect that is usually overlooked in such studies is the potential effect of the width $D$ of the auroral curtain on the brightness and CR retrieved from the observations. These features are accounted for in our auroral model and thoroughly described in Appendix A.1. In brief, our model uses the atmosphere described in Grodent et al. (2001), which accounts for the higher gravity near the poles, the influence of the electron precipitation on the thermal structure, as well as several observational constraints such as the altitude of the $\mathrm{H}_{2}$ emission peak, the temperature profile deduced from UV and infrared emissions, and UV emission rates deduced from observed images and spectra. To relate $\langle E\rangle$ to $\mathrm{CR}$, we used single Maxwellian distributions with $\langle E\rangle$ from 2 to $1000 \mathrm{keV}$, representing a wide range of initial energy distributions of the precipitating electrons. An auroral volume emission rate vertical profile (VER: number of auroral photons emitted per second per $\mathrm{cm}^{3}$ ) is then produced from each Maxwellian distribution with the two-stream Grodent et al. (2001) model. For each primary electron distribution, two synthetic emergent spectra are then calculated for emission angles $\chi$ from $0^{\circ}$ to $80^{\circ}$ and auroral widths of $150 \mathrm{~km}$ (representing the IFP, GFP and SSS), $600 \mathrm{~km}$ (representing the main emissions) and $1500 \mathrm{~km}$ (representing the flare emissions in the polar cap region). A first synthetic spectrum, unaffected by the hydrocarbon attenuation is used to determine the influence of $\langle E\rangle, D$, and $\chi$ on the intrinsic auroral brightness while a second 
synthetic spectrum, absorbed by the hydrocarbons, is used to determine the influence of these parameters on the CR. Results from this modeling work are presented in Fig. 11 in Appendix A.1. It is seen that (1) for a given $\langle E\rangle$, the CR noticeably increases with $\chi,(2)$ for a given $\chi$, the auroral width $D$ has a minor effect on the CR, and (3) the unabsorbed brightness depends strongly on both $\chi$ and $D$.

Additional outputs from this model, described in Appendix A.2, focus on the characteristics of the VER profiles resulting from the different Maxwellian electron distributions. Results, summarized in Fig. 12, show that (1) the altitude of the auroral peak decreases with increasing $\langle E\rangle$ and reaches a minimum value at $\sim 150 \mathrm{~km},(2)$ the FWHM of the VER profile decreases with increasing $\langle E\rangle$ (FWHM $=440 \mathrm{~km}$ for $\langle E\rangle=2 \mathrm{keV}$ and $53 \mathrm{~km}$ for $500 \mathrm{keV}$ ), and (3) for a constant energy input of $1 \mathrm{~mW} \mathrm{~m}^{-2}$, the emergent brightness strongly depends on $\langle E\rangle$, with a maximum value of $14.6 \mathrm{kR}$ at $\langle E\rangle=20 \mathrm{keV}$ and a decrease toward higher energies, reaching $3.2 \mathrm{kR}$ at $\langle E\rangle=500 \mathrm{keV}$.

\subsection{Important remarks regarding the energy-CR relation}

To date, there is no direct observation that strongly constrains:

1. The temperature profile in the polar regions (i.e. the pressurealtitude relationship). The knowledge of the $\mathrm{H}_{2}$ density profile is crucial, as it provides the penetration depth of the precipitating electrons for a given mean energy. For different temperature profiles, a given pressure level will correspond to different altitudes and thus to different levels of absorption. Consequently, the use of, for example, the Grodent et al. (2001) or the Moses et al. (2005) temperature profile does not yield the same $\langle E\rangle-$ $\mathrm{CR}$ relationship. Also, the temperature profile affects the scale height of the emission and thus the shape of the auroral vertical profile, which influences the portion of the aurora affected by hydrocarbons.

2. The altitude of the hydrocarbon homopause, which influences the level of absorption for a given altitude of auroral emission. In particular, the eddy diffusion coefficient $K_{h}$ controls the hydrocarbon mixing strength in the homosphere and thus plays an important role in the relative hydrocarbon absorption of the auroral emission for a given energy distribution of electrons.

3. The energy distribution of the precipitating electrons, which strongly affects the shape and altitude of the VER profile of the auroral emission. For example, a monokinetic beam of $100 \mathrm{keV}$ electrons produces a vertical $\mathrm{H}_{2}$ emission with $\mathrm{CR}=3.9$ while a Maxwellian distribution with $\langle E\rangle=100 \mathrm{keV}$ produces a vertical emission with $\mathrm{CR}=3.0$. The type of electron energy distributions thus influences the mean energy inferred from a given $C R$. In the case of kappa distributions, which are controlled by two parameters (the most probable value $E_{0}$ and the spectra index $k$ that controls the amplitude of the distribution toward high energies), the $\langle E\rangle-C R$ relationship is not unique, as two distributions with same $\langle E\rangle$ may lead to VER of different shape and thus of different color ratios.

As the $\mathrm{CR}-\langle E\rangle$ relationship depends on unconstrained auroral characteristics, it is strongly model-dependent. In this work we chose the auroral atmosphere described by Grodent et al. (2001), which is the most appropriate for auroral studies, and used single Maxwellian distributions to create a $\langle E\rangle-C R$ relationship. The nominal eddy diffusion coefficient in Grodent et al. (2001) is $K_{h}=1.4$ $\times 10^{6} \mathrm{~cm}^{2} \mathrm{~s}^{-1}$ at the homopause. Assuming a doubling of the $K_{h}$ coefficient, we estimate the error on the energy determinations as approximately $30 \%$ of the nominal value. This value $(30 \%)$ should be seen as a lower estimate of the total error, as other sources of uncertainty occur. These include errors propagated by data manipulations (such as background subtraction, ...), or uncertainties on the calibration curves and various cross-section measurements needed in this study.

Constraints on the parameters just described could be provided by (1) solar or stellar occultations (for the temperature profile and pressure-altitude relationship), (2) direct observation of the potential drop in the acceleration region and the electron energy distribution, and (3) direct observation of the altitudinal UV profile from images, from which the electron energy distribution could be inferred. The latter point is important, as VER profiles of the IFP have been observed from ACS images and analyzed by Bonfond et al. (2009). This special case will be discussed in Section 4.3.4. The observations that should be made with the different instruments onboard the Juno spacecraft, from mid-2016 to late 2017 are expected to significantly contribute to these aspects of our understanding of the jovian aurora.

In the following analysis, three different topics have been addressed. First, the auroral model briefly described in the preceding lines is directly applied to the spectral images to provide spatial maps of the electron energy. Several auroral regions have been delimited and results are discussed in Section 4.1. Second, the energy-energy flux relationship for the different auroral regions is examined in order to infer the processes leading to these emissions (Section 4.2). Third, the signal in the pixels forming the different auroral regions has been summed to provide corresponding average spectra. Similarly, new electron energy distributions have been determined for each region. This analysis is provided in Section 4.3.

\section{Auroral characteristics inferred from observed spectral images}

\subsection{Map of the auroral electron energy}

The first application of the model described in Section 3.1 and Appendix $A$ is the determination of the mean energy $\langle E\rangle$ of the precipitating electrons from the two STIS reconstructed images, whose spatial pixels contain spectral information. First, a map of the CR is obtained from the ratio between the images integrated in the (1550-1620) $\AA$ and (1230-1300) Å bandwidths. Second, a map of angle $\chi$ is calculated for each spatial pixel from latitude and longitude grids as described in Section 2. Values of CR and $\chi$ are thus associated with each spatial pixel, from which $\langle E\rangle$ is determined, based on interpolation of the CR- $\langle E\rangle$ relationship obtained at various $\chi$ angles (see Fig. 11e). Fig. 2 shows the map of the precipitating electron mean energies $\langle E\rangle$ for the two observations. The different auroral regions defined in Fig. 1 are also outlined in Fig. 2. A more detailed view of the dispersion of the brightness and mean energies within each region is obtained by grouping the brightness and $\langle E\rangle$ of each pixel by bins of 5 or $10 \mathrm{kR}$ (or $\mathrm{keV}$ ), forming histograms of the vertical brightness and mean energy, as shown in Fig. 3 and Table 1. Each point included in these histograms corresponds to the brightness and mean energy of a spatial pixel of the auroral regions considered. In order to remove the effects of viewing angle, usually not considered in auroral models, the observed brightnesses have been converted to vertical brightness for each pixel, following the curve plotted in Fig. 11c. The moderate influence of $\langle E\rangle$ on this conversion has also been taken into account in the calculations. For the main emissions (ME region 1 and ME region 2) and discontinuity (DC, region 5), we assume an auroral width of $600 \mathrm{~km}$. For the flares (FL, region 3 and 4) we assume a width of $1500 \mathrm{~km}$. A width of $150 \mathrm{~km}$ is then assumed for the Io footprint (IFP, region 6 ), the small scale structure (SSS, region 7 in obs. \#1) and the Ganymede footprint (GFP, region 7 in obs. \#2). 


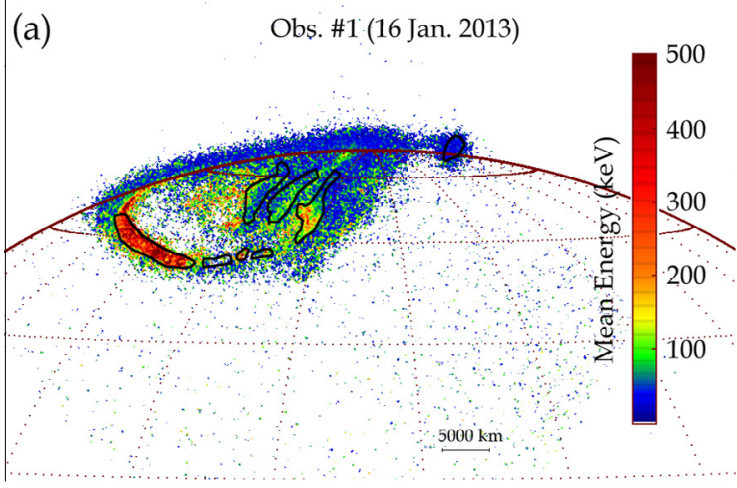

(c)
Obs. \#2 (24 Jan. 2013)

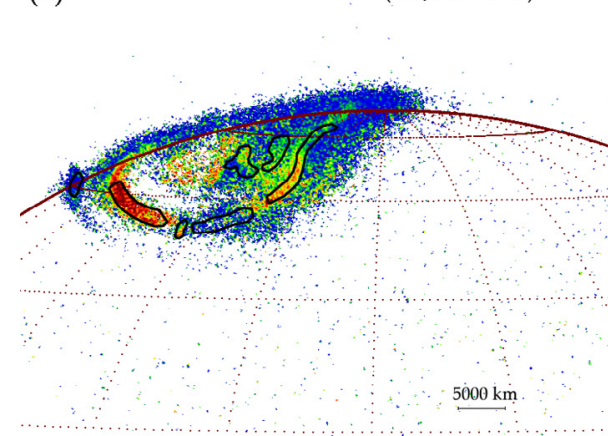

(b)

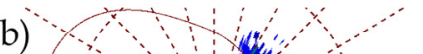

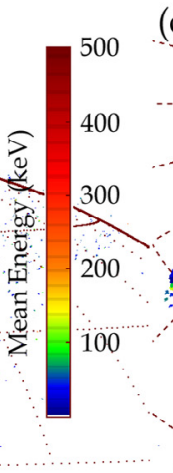

(d)

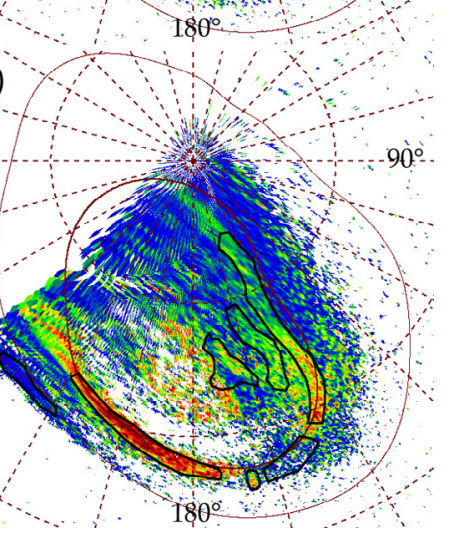

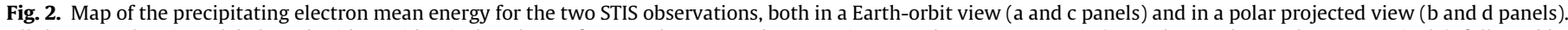

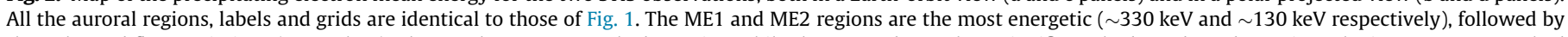

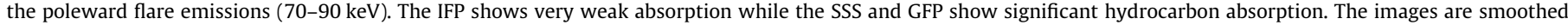
over a 3 pixels boxcar for better legibility.

In order to empirically determine the brightness and mean energy distribution associated with each auroral region, both the vertical brightness and $\langle E\rangle$ histograms have been fitted with two distributions often used in this field of study. First, a Maxwellian distribution,

$D=C \frac{E}{E_{0}} e^{-E / E_{0}}$,

and a kappa distribution

$D=C \frac{E}{E_{0}}\left(1+E \frac{E_{0}}{k}\right) e^{(-1-k)}$.

A third distribution, allowing a greater flexibility in terms of shape, has also been used, namely the 'generalized' Maxwellian distribution

$D=C E^{n / 2} E_{0}^{-\left(\frac{n}{2}+1\right)} e^{-E / E_{0}}$.

In these equations, $C$ is a constant factor, $E$ is the electron energy (or the brightness), $E_{0}$ is the most probable value for the Maxwellian and kappa distributions (i.e. the characteristic energy), while the most probable value is $n E_{0} / 2$ for the generalized Maxwellian. Parameter $k$ is the spectral index of the kappa distribution and $n$ is a free parameter of the generalized Maxwellian distribution. This distribution is Maxwellian for $n=1$ and becomes strongly nonMaxwellian for high values of $n$. More details on this distribution may be found in Dzifčáková (1998) and references therein. Fig. 3 shows that the kappa and Maxwellian distributions do not provide good fits to either the brightness or energy histograms of the different regions. Instead, the generalized Maxwellian provides the best agreement with the observed profiles, usually with strong nonMaxwellian characteristics $(n \gg 1)$. Interestingly, the brightness and energy distribution of each auroral zone share very similar characteristics for both observations. The $E_{0}$ and $n$ parameters of the best generalized Maxwellian are provided in Table 1 .

As seen in Fig. 3a, b, o and p, the ME 1 region is very bright and characterized by the most energetic primary electrons. The vertical brightness distributions are relatively symmetric, around $\sim 260 \mathrm{kR}$. The electron mean energies $\langle E\rangle$ determined from the CR method are mostly between 100 and $700 \mathrm{keV}$, with several individual pixels at higher or lower values. The average $\langle E\rangle$ value is quite high ( $\sim 300$ and $\sim 355 \mathrm{keV}$ for observation 1 and 2, respectively). It should be noted that mean energies found in the literature are generally determined from spectra obtained from the sum of numerous pixels, which averages the energies so obtained, while the distributions shown here reflect the range of values reached by the individual pixels of the auroral region, which expand the range of inferred values. Fig. 30 and p clearly show that several pixels have $\langle E\rangle$ larger than $400 \mathrm{keV}$, well above values generally observed. As seen in Fig. 11a, for $\langle E\rangle$ larger than $400 \mathrm{keV}$, the CR is larger than 20 for a viewing angle of $70^{\circ}$. For a pixel with a total unabsorbed UV brightness of $200 \mathrm{kR}$ and $\mathrm{CR}=20$, the $(1550-1620 \AA)$ unabsorbed intensity is $25 \mathrm{kR}$, while the absorbed (1230-1300 $\AA$ ) emission is 20 times less, i.e. $1.25 \mathrm{kR}$. In other words, pixels associated with very high electron energy undergo a very low $\mathrm{S} / \mathrm{N}$ in the absorbed spectral bandwidth, which induces a very high uncertainty on the energies inferred for high energy pixels of the distributions. As a result of this large uncertainty, pixels with $\langle E\rangle$ larger than $400 \mathrm{keV}$ should be considered with caution. A way to overcome this problem is to bin the pixels in groups or sum all the pixels of a given region, to increase the $\mathrm{S} / \mathrm{N}$ ratio, as proposed in Sections 4.2.1 and 4.3.1. The mean energy map (Fig. 2) and distributions (Fig. 3) presented here should then be seen as first order estimations.

The bright winding afternoon main emission (ME 2 "kink" region) shows a very asymmetrical vertical brightness distribution, 

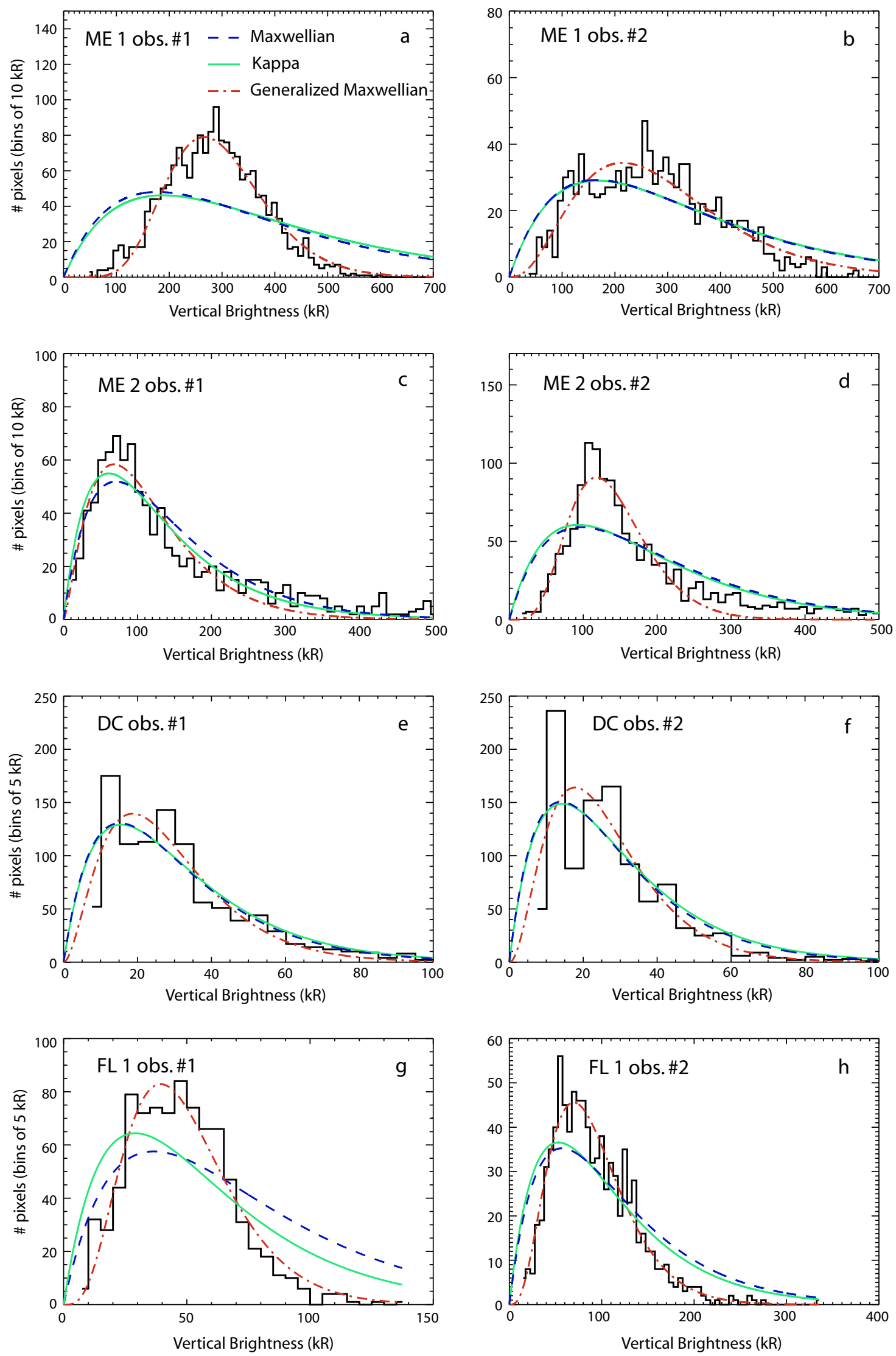

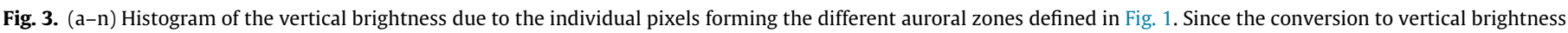

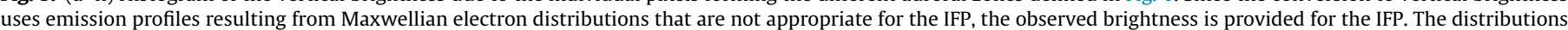

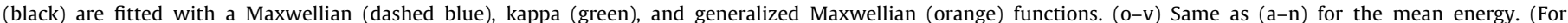
interpretation of the references to color in this figure legend, the reader is referred to the web version of this article.) 

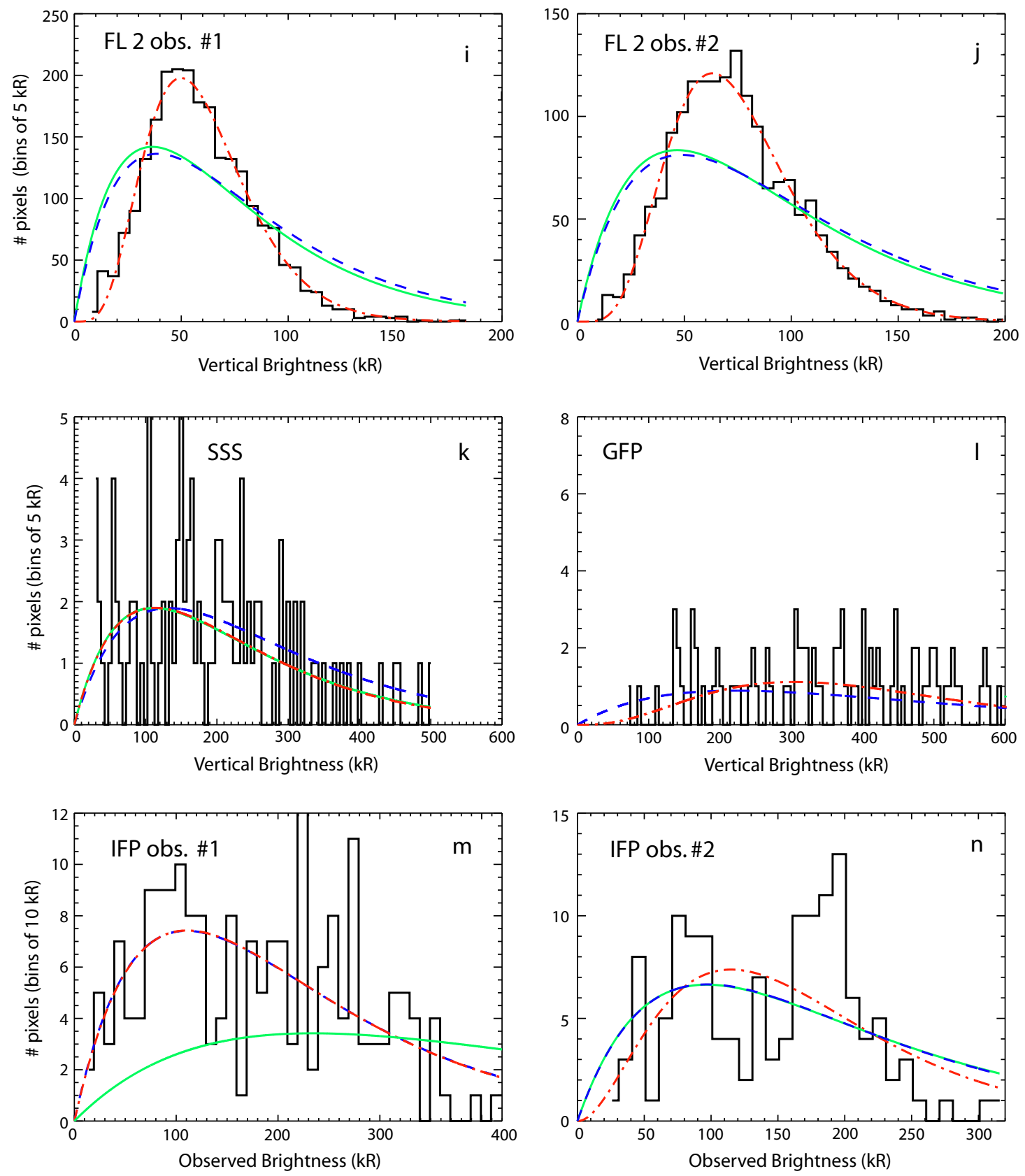

Fig. 3 (continued)

with an average value around $160 \mathrm{kR}$, reaching values above $400 \mathrm{kR}$ for several pixels. Although the mean energies are large $(\sim 130 \mathrm{keV}),\langle E\rangle$ in ME 2 are roughly 2.5 times lower than ME 1 values. The distributions are still best fitted by the generalized Maxwellian function.

The vertical brightness distributions from the four polar cap emissions selected in region 3 and 4 (FL 1 and FL 2) are very comparable in shape and value, with peaks between 40 and $70 \mathrm{kR}$. The mean energies are significantly lower than in the main emission, with average values between $\sim 65$ and $\sim 100 \mathrm{keV}$. Again, the departure from a Maxwellian distribution is clear. The $\langle E\rangle$ distributions are relatively narrow with FWHM $\sim 80 \mathrm{keV}$, and with virtually no electrons with energies $>200 \mathrm{keV}$.

As expected, the discontinuity region exhibits very weak emission, with an average at $\sim 30 \mathrm{kR}$. The three tested distributions provide a reasonable fit to the brightness histogram, again with a best model provided by the generalized Maxwellian. In terms of mean energies, a substantial number of individual pixels ( $\sim 40 \%)$ have a total UV intensity lower than $25 \mathrm{kR}$ (thus low $\mathrm{S} / \mathrm{N}$ ), which does not allow a safe determination of $\langle E\rangle$. We thus estimate that the energy distributions from individual pixels in the discontinuity region are not trustworthy and are disregarded from this analysis. A precise determination of $\langle E\rangle$ will be discussed in the next section, based on the spectra obtained from the summed pixels of this region.

The number of pixels belonging to the Io footprint is relatively small (198 for observation \#1 and 138 for observation \#2), which makes it difficult to derive statistically significant distributions. In addition, the CR method is probably not appropriate to determine $\langle E\rangle$ for each pixel, because (1) the CR from our data is always close to the unabsorbed value, which makes $\langle E\rangle$ more sensitive to $\mathrm{S} / \mathrm{N}$ ratio, and (2) the Maxwellian distributions used in the CR method 

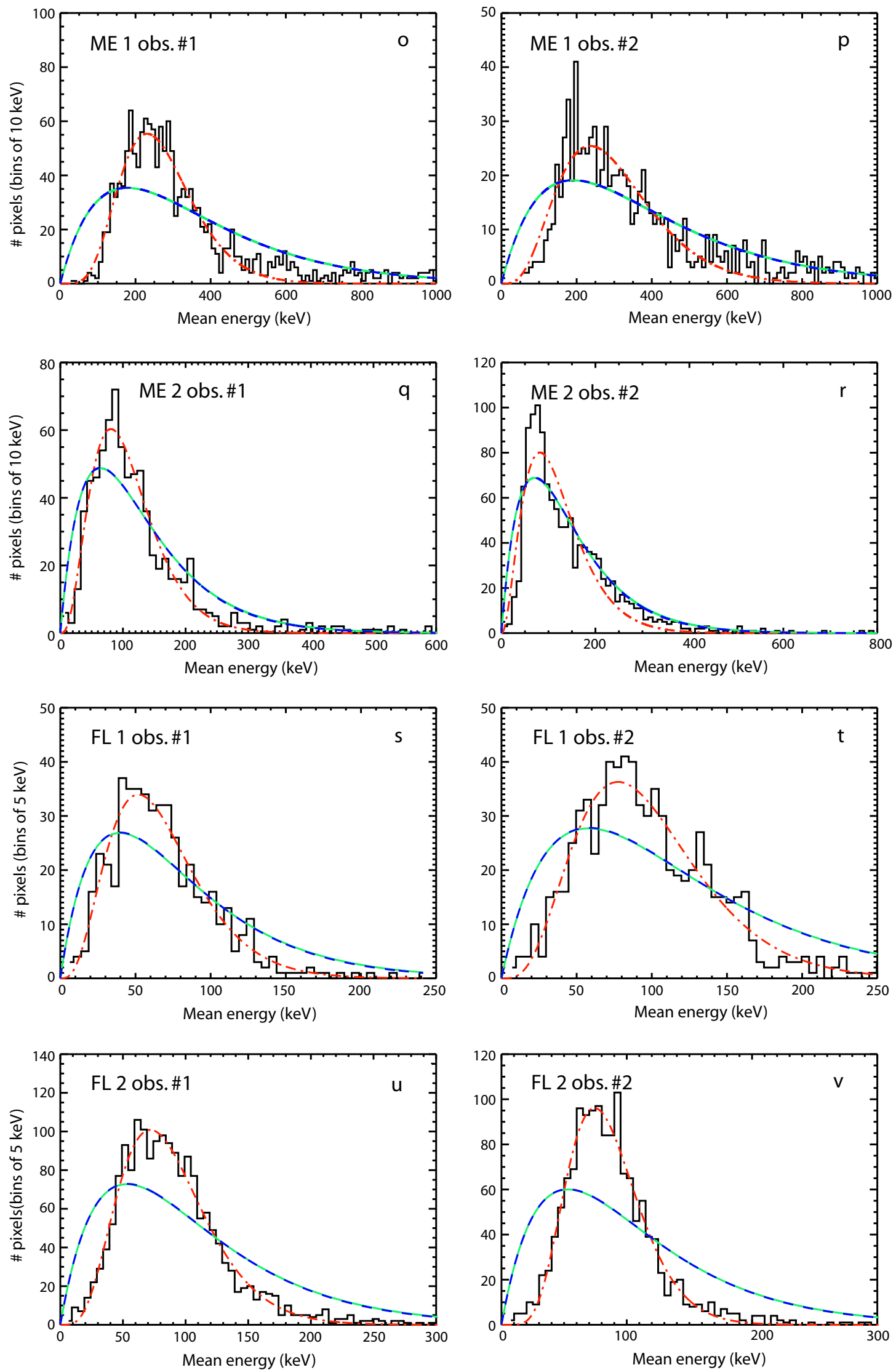

Fig. 3 (continued) 
Table 1

Brightness and mean energy of the different auroral regions shown in Figs. 1 and 2.

\begin{tabular}{|c|c|c|c|c|c|c|c|c|c|c|c|c|c|c|}
\hline & \multicolumn{7}{|c|}{ Observation \#1 (16 Jan. 2013) } & \multicolumn{7}{|c|}{ Observation \#2 (24 Jan. 2013) } \\
\hline & ME 1 & ME 2 & FL 1 & FL 2 & DC & IFP & SSS & ME 1 & ME 2 & FL 1 & FL 2 & DC & IFP & GFP \\
\hline \# pixels & 1801 & 988 & 865 & 2350 & 1319 & 198 & 112 & 1107 & 1381 & 905 & 1725 & 1314 & 138 & 95 \\
\hline Mean view angle $\left(^{\circ}\right)$ & 69 & 66 & 72 & 69 & 59 & 80 & 60 & 66 & 67 & 68 & 69 & 58 & 76 & 58 \\
\hline $\begin{array}{l}\text { Brightness } \\
\text { Mean vertical brightness from images }(k R)^{a} \\
\text { Best fit parameters }{ }^{c}\end{array}$ & 286 & 148 & 47 & 59 & 31 & $178^{\mathrm{b}}$ & 194 & 273 & 180 & 91 & 75 & 28 & $145^{\mathrm{b}}$ & 361 \\
\hline$E_{0}$ & 30.5 & 48.1 & 10.5 & 10.6 & 10.0 & 109.0 & 113.1 & 78.5 & 21.7 & 21.4 & 12.2 & 8.3 & 55.7 & 102.7 \\
\hline$n$ & 17.5 & 2.8 & 7.5 & 9.4 & 3.7 & 2.0 & 2.0 & 5.5 & 10.9 & 6.5 & 10.3 & 4.3 & 4.1 & 5.9 \\
\hline Vertical brightness from spectrum $(\mathrm{kR})^{\mathrm{a}, \mathrm{d}}$ & 219 & 132 & 48 & 58 & 36 & - & 91 & 215 & 166 & 88 & 73 & 17 & - & 242 \\
\hline Observed brightness from spectrum $(\mathrm{kR})^{\mathrm{a}}$ & 278 & 204 & 79 & 93 & 32 & 175 & 57 & 259 & 264 & 140 & 118 & 15 & 144 & 109 \\
\hline $\begin{array}{l}\text { Mean energy } \\
\text { Mean observed value from image }(\mathrm{keV})^{\mathrm{e}} \\
\text { Best fit parameters }^{\mathrm{c}}\end{array}$ & 301 & 122 & 69 & 91 & - & - & - & 356 & 138 & 69 & 88 & - & - & - \\
\hline$E_{0}$ & 38.6 & 27.4 & 15.7 & 16.1 & - & - & - & 61.5 & 38.2 & 20.6 & 10.7 & - & - & - \\
\hline$n$ & 12.0 & 5.9 & 6.6 & 8.9 & - & - & - & 7.7 & 4.3 & 7.5 & 13.9 & - & - & - \\
\hline CR from spectrum & 13.6 & 3.9 & 2.0 & 2.9 & 2.3 & 1.3 & 11.0 & 13.0 & 4.4 & 3.2 & 2.9 & 1.9 & 1.3 & 6.7 \\
\hline Value from spectrum $(\mathrm{keV})^{\mathrm{e}}$ & 278 & 102 & 52 & 77 & 67 & - & 232 & 259 & 111 & 86 & 79 & 53 & - & 157 \\
\hline Value from constrained distribution $(\mathrm{keV})^{\mathrm{f}}$ & 256 & 106 & 51 & 71 & - & - & - & 275 & 112 & 83 & 78 & - & - & - \\
\hline
\end{tabular}

${ }^{a}$ Unabsorbed brightness in 700-1700 Å derived from maps shown in Fig. 1.

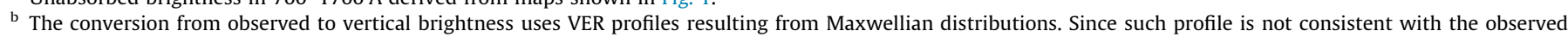
constraints in the case of the IFP, observed values are given for the IFP emission.

c Best parameters of the generalized Maxwellian distribution for histograms based on all pixels included in each auroral region (see Fig. 3).

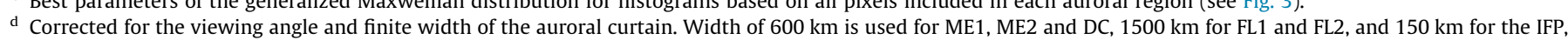
GFP and small scale structure.

e Based on the CR method.

f See Section 4.3.3.

to link $\langle E\rangle$ to CR are probably not appropriate in the IFP case. Indeed, from several IFP emission profiles determined from ACS images, Bonfond et al. (2009) determined an average VER consistent with precipitating electrons in a kappa distribution instead of a Maxwellian, which modifies the CR- $\langle E\rangle$ relationship (see Section 3.2). This point will be discussed in more details in the spectral analysis (Section 4.3.4). Consequently, the distribution of the pixels per energy bin cannot be determined for the IFP. Our sample shows observed brightnesses from 15 to $420 \mathrm{kR}$. Due to the effect of viewing angle and auroral width (assumed to be $150 \mathrm{~km}$ for footprints in the model), these observed values should be converted to vertical values. Again, this conversion is not possible because of the unknown VER profile of each individual pixel. We thus decided to show in Fig. 3 the observed IFP brightness distribution instead of the vertical value. Because of the very narrow range of viewing angles associated with the IFP spot, the shape of the vertical and observed brightness distributions are virtually identical. The only difference is the absolute value of the brightness, which thus shifts the whole brightness distribution accordingly. The best Maxwellian electron distribution used by Bonfond et al. (2009) to fit the average IFP VER profile has a mean energy of $2 \mathrm{keV}$. Although this Maxwellian distribution does not provide the best fit the IFP VER profile, it allows us to estimate the observed-to-vertical conversion factor from our model. With such energy and a viewing angle of $80^{\circ}$, the observed brightness must be multiplied by 7 to represent the vertical value, which is consistent with vertical conversion factors determined by Bonfond et al. (2013) from 3D simulations. This quite big conversion factor is explained by the very limited extent of the intrinsic IFP auroral emission, which thus needs to be very intense to provide the observed values. The vertical brightness distribution of our sample thus extends from $\sim 100$ to $\sim 3000 \mathrm{kR}$.

The small scale structure (SSS) is also clearly seen in the 16 Jan. 2013 observation (region 7 in Fig. 1a and b). Since it is situated inside the discontinuity region, we specifically used the discontinuity emission as background for the SSS in order to withdraw the potential influences of the DC on the SSS emission. Because of the relatively low number of pixels in the SSS region $(\sim 100$, to be compared with values between 850 and 2400 for the main emission and flare regions), the brightness distributions, spread over the 30-400 kR range, do not reveal a clear shape. We assumed an auroral curtain of width $150 \mathrm{~km}$ to convert observed to vertical brightnesses, which corresponds to a factor of $\sim 2$ for $\langle E\rangle=200 \mathrm{keV}$. As for the IFP, the low number of pixels considered does not allow us to present a reliable distribution of mean energies. Still, a first order examination of the mean energy distributions shows that the average $\langle E\rangle$ is surprisingly large, at $\sim 260 \mathrm{keV}$. Again, a more precise examination of SSS characteristics, based on the spectra obtained from the sum of all SSS pixels is discussed in Section 4.3.5. Characteristics of the GFP emission (region 7 in Fig. 1c and d) are very close to those of the SSS. The low number of pixels defining the GFP makes it difficult to infer a mean energy from the distribution, but the average value is around $120 \mathrm{keV}$.

\subsection{Mean electron energy-energy flux relationship}

\subsubsection{Determination of the data points}

After the first-order overview based on the STIS maps (Figs. 1 and 2) and individual pixel brightness and energy distributions (Fig. 3), a more accurate examination of each region's characteristics is given in this section. As already mentioned, $\langle E\rangle$ may be loosely determined when the individual pixels exhibit a low count rate (hence low $\mathrm{S} / \mathrm{N}$ ), which consequently increases the uncertainty of the $\langle E\rangle$ retrievals. To improve the accuracy of the mean energy and energy flux values (and thus avoid large scatter of the data points in a mean electron energy-energy flux diagram), we sorted the pixels belonging to each zone from lowest to highest brightness, and binned them by groups of eight pixels. We thus implicitly assume that there is an unequivocal relationship between the energy flux and the mean energy. A higher $\mathrm{S} / \mathrm{N}$ spectrum is derived from each new point, from which a new $C R$ hence $\langle E\rangle$ is determined using the CR method described in the previous section. The energy flux is determined for each new point following the relationship illustrated in Fig. $12 \mathrm{~d}$, where the brightnessenergy flux conversion is energy-dependent. This method is much better than a simple smoothing or binning of the original data points, as it removes potential unreliable values from original 
low $\mathrm{S} / \mathrm{N}$ pixels. As expected, the mean energy-energy flux diagrams derived from the binned data points conserve the trend obtained from the original pixels, but with much less dispersion. Very high localized mean energies seen in some distributions in the previous section (for example, $\langle E\rangle$ above $400 \mathrm{keV}$ for ME 1 in Fig. 3a) disappear in the newly binned energy data points.

The main remaining source of uncertainty in the mean electron energy determination with the CR method stems from the hydrocarbon density profiles, not constrained by observations in the polar regions. As described by Gustin et al. (2004a), we determined the uncertainty in $\langle E\rangle$ by considering the effect of doubling the eddy diffusion coefficient $K_{h}$, which increases the homopause altitude. This coefficient is $1.4 \times 10^{6} \mathrm{~cm}^{2} \mathrm{~s}^{-1}$ in the nominal atmospheric model we use. This variation of the homopause altitude has a non-linear effect on the CR and is thus energy dependent, which implies a $\sim 12 \%$ to $30 \%$ uncertainty in $\langle E\rangle$. The uncertainty on the energy flux comes from the $\mathrm{S} / \mathrm{N}$ ratio (error between $\sim 5 \%$ and $\sim 25 \%$ ) and the systematic uncertainty due to the $\mathrm{kR}-\mathrm{mW} \mathrm{m}^{-2}$ conversion factor, which is model dependent. At $100 \mathrm{keV}$, the latter factor may vary from 8.4 to $10.6 \mathrm{kR}$ per injected $\mathrm{mW} \mathrm{m}^{-2}$ depending on the atmospheric model and method chosen, which represents a constant $22 \%$ uncertainty. The error in the energy flux thus varies from $\sim 23 \%$ to $33 \%$.

\subsubsection{Theory}

The relationship between the energy fluxes and precipitation energies inferred from the data can be compared to theoretical predictions for static auroral acceleration. Based on Knight's (1973) kinetic theory of field-aligned currents, Lundin and Sandahl (1978) first derived the relationship between the incident energy flux at the atmosphere and the energy of electrons precipitating through a steady-state, monotonic field-aligned potential drop. The location and extent of the field-aligned potential was specified by the mirror ratio, $R_{x}=B_{i} / B_{x}$, between the top of the acceleration region, denoted by the subscript $x$, and the atmosphere, denoted by the subscript $i$. An isotropic, Maxwellian distribution of electrons was assumed at the top of the acceleration region. The precipitating electron energy flux is then described by

$$
\begin{aligned}
E_{f i}= & n_{x}\left(\frac{W_{x}}{2 \pi m_{e}}\right)^{1 / 2}\left[R_{x}\left(2 W_{x}+e \Phi_{\|}\right)-\left(R_{x} e \Phi_{\|}\right.\right. \\
& \left.\left.+2\left(R_{x}-1\right) W_{x}\right) \exp \left(-\frac{e \Phi_{\|}}{W_{x}\left(R_{x}-1\right)}\right)\right],
\end{aligned}
$$

where $W_{x}$ is the electron temperature (in energy units) at the top of the acceleration region, $\Phi_{\|}$is the magnitude of the potential drop, $m_{e}$ is the mass of the electron, $n_{x}$ is the electron density at the top of the acceleration region, and $e$ is the fundamental charge of the electron. Lundin and Sandahl (1978) also showed that in the limit where $e \Phi_{\|} \gg W_{x}$ and $B_{i} \gg B_{x}$, the relationship can be simplified to

$E_{f i}=n_{x} \sqrt{\frac{1}{2 \pi m_{e}}} W_{x}^{-1 / 2}\left(e \Phi_{\|}\right)^{2}$.

Physically, this limit describes an accelerating potential in which the energy gained by the electron is far greater than its initial energy, and the extent of the potential drop is such that an infinite reservoir of electrons can be assumed at the top of the acceleration region.

Jupiter's main auroral emission is, to first order, a steady-state phenomenon that is driven by the outward transport of Iogenic plasma within the magnetosphere and fixed in the frame rotating with the planet. Therefore, the steady-state relationship between the energy flux and field-aligned potential strength described by Eq. (4) is applicable and, when combined with the mean energy and energy flux of the precipitating electrons inferred from STIS observations, can offer insight into the properties of the auroral acceleration region and the source population of the precipitating electrons. The location of the acceleration region, and hence whether or not Eq. (5) is applicable for the jovian system has not yet been determined. This has been discussed in detail (e.g. Cowley and Bunce, 2003; Su et al., 2003; Nichols and Cowley, 2005; Ray et al., 2009, 2010) and will be explored further in a companion paper (Ray et al., in preparation).

The Lundin and Sandahl (1978) analysis was for the terrestrial system, where the precipitating electron energies are on the order of $10 \mathrm{~s}$ of $\mathrm{keV}$. At Jupiter, the inferred precipitating energies are much larger, sometimes reaching $500 \mathrm{keV}$. At these magnitudes, the precipitating electrons are relativistic. Cowley (2006) derived current-voltage and energy flux relations for an isotropic Maxwellian distribution of electrons accelerated by field-aligned potentials to relativistic speeds. The kinetic energy flux deposited in the planetary atmosphere by relativistic electrons can exceed that estimated by the non-relativistic relation by a factor of 2 (for $e \Phi_{\|} /$ $k W_{x}=20, W_{x}=25 \mathrm{keV}$ ) to 5 (for $e \Phi_{\|} / k W_{x}=200, W_{x}=25 \mathrm{keV}$ ). Therefore, the estimates of the precipitating electron density into the atmosphere will also be modified for a given electron energy flux and precipitating electron energy.

\subsubsection{Analysis}

Fig. 4a shows the energy flux-precipitation energy relationships from the main emission and flare regions of the two STIS observations. What is evident is that the range of energy fluxes and mean energies for the flare (black and orange crosses) and main emission 2 (green stars) regions are coincident, while the mean energies estimated for the main emission 1 regions (light blue stars) are a factor of 2-3 larger. First, we use Eq. (5) to estimate the density of the 'source' magnetospheric electron population, as in Gustin et al. (2004a). Fig. 4b-g shows the individual ME 1 and 2 auroral regions, with the dashed and dashed-dot lines corresponding to the energy flux-mean energy curves for $W_{x}=2.5 \mathrm{keV}$ and densities of $n_{x}=1000 \mathrm{~m}^{-3}$ and $n_{x}=10,000 \mathrm{~m}^{-3}$, respectively. The latter values correspond to the hot electron density range measured near the current sheet by Voyager 1 and 2 (Scudder et al., 1981).

We first estimate the density of the electrons in the middle magnetosphere plasma sheet by determining the best fit to the data using Eq. (5) and $W_{x}=2.5 \mathrm{keV}$ (red and green lines in Fig. 4). The inferred precipitating electron densities for the ME 2 region are $\sim 2400 \mathrm{~m}^{-3}$, consistent with Voyager values. The density estimates from the narrow ME 1 emission are a factor of 3 lower $\left(\sim 800 \mathrm{~m}^{-3}\right)$. However, these emissions map to the dawn or possibly midnight sector of the magnetosphere, which Voyager did not sample. For ME 1, the relativistic energy flux-mean energy relationship has also been tested, as the electron's acceleration is a significant fraction of the electron rest energy for this region (panel 4 $\mathrm{d}$ and e). Compared to the non-relativistic case, electron densities are $\sim 45 \%$ lower, with values of 619 and $521 \mathrm{~m}^{-3}$. These values correspond well with densities obtained from the study of a remarkably bright auroral morning arc observed by STIS on 21 September 1999, which also exhibit very high electron energies (Gustin et al., 2006). It should be noted that the $2.5 \mathrm{keV}$ electron temperature we use is the center energy of the narrow $2-3 \mathrm{keV}$ range quoted by Scudder et al. (1981). Globally, an equivalent Knight curve is obtained from an increase (decrease) of $0.5 \mathrm{keV}$ of the electron temperature associated with an increase (decrease) of the electron density by $\sim 10 \%$.

Unlike the relatively well-understood main aurora emission, it is less clear what acceleration processes drive the variable flare emissions, which map to the outer magnetosphere, or possibly to the boundary region or beyond. Therefore, the energy-energy flux relationship of the flare regions shown in Fig. 4a are not further 

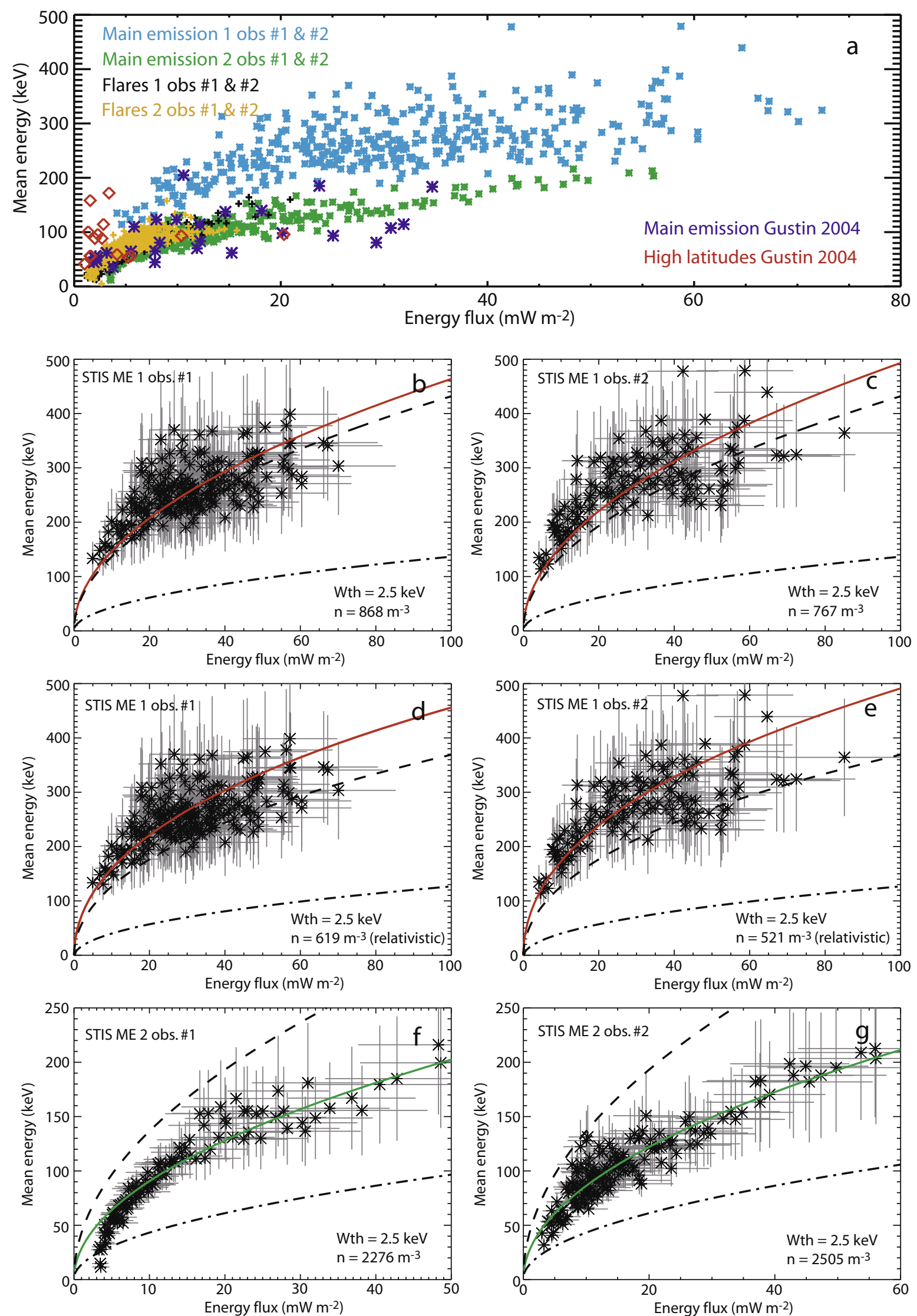

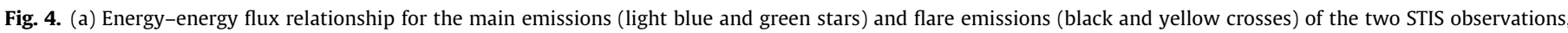

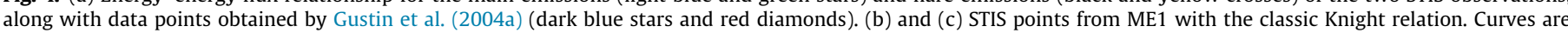

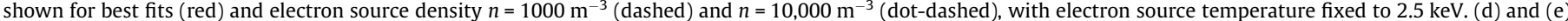

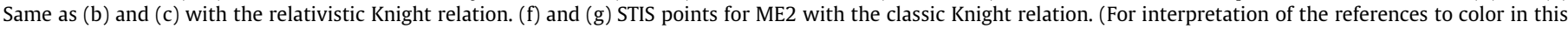
figure legend, the reader is referred to the web version of this article.) 
discussed in the present paper but will be examined in Ray et al., in preparation.

\subsection{Spectral analysis}

\subsubsection{Mean energy from average spectra}

In order to maximize the $\mathrm{S} / \mathrm{N}$ of the spectral information at each auroral region defined in Fig. 1, an average spectrum has been constructed for each region by summing the spectra associated with the spatial pixels defining each auroral region. Since each spectrum may correspond to a region that covers substantial latitude and longitude ranges, an average viewing angle has been determined in each case. In contrast to the average background used for the $\langle E\rangle$ map production shown in Fig. 2, a specific background, chosen close to the auroral region considered, has been used and subtracted to each spectrum. The mean energy of the extracted spectra, determined from the CR method, and the unabsorbed UV brightness in the 700-1700 A range are summarized in Table 1. For a given region, values from the average spectrum and the distributions described in Section 4.1 are slightly different. This is explained by several factors, such as the different background subtraction, the average viewing angle associated with each spectrum, the lower $\mathrm{S} / \mathrm{N}$ ratio of the individual pixels used to determine the mean energy of each pixel, etc.

\subsubsection{Can the energy distribution be inferred from spectral analysis?}

As mentioned before, since no observation provides sufficient constraints on the energy distribution of the precipitating electrons and on the auroral VER profiles, the $\langle E\rangle-\mathrm{CR}$ relationship is not unequivocal: different electron energy distributions with the same $\langle E\rangle$ will produce emergent spectra with different color ratios. Our CR method includes the contributions of $\mathrm{CH}_{4}, \mathrm{C}_{2} \mathrm{H}_{2}$ and $\mathrm{C}_{2} \mathrm{H}_{6}$ to attenuate the auroral signal, but only accounts for the absorbed 1230-1300 Å spectral range. As we reach deeper atmospheric layers $(\sim 250 \mathrm{~km})$, the density profiles of acetylene and ethane become very important and affect the shape of the auroral spectra, especially in the 1330-1530 A spectral regions, not used in the CR definition. Indeed, unlike $\mathrm{CH}_{4}$, density profiles of $\mathrm{C}_{2} \mathrm{H}_{2}$ and $\mathrm{C}_{2} \mathrm{H}_{6}$ do not monotonically increase with decreasing altitude, instead presenting density peaks (see Fig. 9c). Since the amount of absorption depends on the altitude of the emission but also on the shape of the VER profile, specific absorption of $\mathrm{C}_{2} \mathrm{H}_{2}$ and $\mathrm{C}_{2} \mathrm{H}_{6}$ may bring new constraints on the VER. Here we thus test the possibility of determining a VER profile that would provide a fit to a given observed spectrum that is significantly better than any other VER. This preferred VER could then be used to infer the 'best' electron energy distribution responsible for this emission.

We use synthetic spectra produced by the method described in Appendix A.1 to fit the observed spectra. At $\sim 12 \AA$ spectral resolution, the specific signature of each hydrocarbon can be easily identified. We used Chapman profiles to simulate the VER, using the parameters described by Eq. (10) in Appendix A.2 as free parameters. The unabsorbed $\mathrm{H}_{2}$ spectrum generated at each altitude bin of the synthetic VER profile is absorbed by the overlying hydrocarbon layer, and a total emergent (absorbed) spectrum is calculated using the atmospheric model described in Section 3.1 and Appendix A.1, taking into account the finite width of the auroral curtain and the mean viewing angle of the auroral region considered. The synthetic emergent spectrum is compared to the observed spectrum and the VER parameters varied until the difference between the two spectra reaches a minimum chi-square. This method thus presumes that a unique VER profile provides a spectral fit that is significantly better than any other synthetic VER profiles. This method has been tested on the spectra of all auroral regions and is shown here for the STIS ME 1 spectrum of obs. \#1, which is strongly attenuated by hydrocarbons $(C R=13.6)$. This attenuation implies that the auroral emission reaches altitudes where $\mathrm{C}_{2} \mathrm{H}_{2}$ and $\mathrm{C}_{2} \mathrm{H}_{6}$ number densities are important enough to induce clear signatures of absorption, hence stronger constraints on the fitting procedure. Results from this spectral analysis demonstrate that several synthetic VERs provide synthetic spectra that satisfactorily fit the ME 1 spectrum, which makes it impossible to unequivocally determine electron characteristics from spectral analysis. Fig. 5 shows two simulations (plain red and green curves) where the synthetic VERs, although quite different, lead to very similar fits to ME 1 spectrum. As seen in Fig. 5a, the VER profile from fit 1 (red) is more intense, peaks at lower altitude, and has a smaller FWHM than VER from fit 2 (green). The corresponding emergent spectra (red and green curves in Fig. 5b and c), are difficult to distinguish from the data and do not allow one to select a preferred model. The light blue dashed curve in Fig. 5b and c shows the unabsorbed emergent spectrum and gives an idea of the hydrocarbon attenuation. It should be noted that a one-layer model (an emitted layer overlaid by an absorbing layer) was tested too and never provided a satisfactory fit to the data, thus supporting a more complex approach such as the one described here. We used Fig. 12a to determine $\langle E\rangle$ from the peak altitude of the synthetic VER. The best VER 1 peaks at $165 \mathrm{~km}$, which corresponds to $375 \mathrm{keV}$. By contrast, VER 2 peaks at $195 \mathrm{~km}$ and corresponds to $\langle E\rangle=188 \mathrm{keV}$. These two energies thus lie either side of the $278 \mathrm{keV}$ value obtained from the CR method. This example demonstrates that the $\langle E\rangle$ value and thus the electron distribution cannot be determined from a spectral analysis without additional constraints. The CR method thus provides a fair estimate of the energy of the electrons responsible for the auroral emission, under the limits provided by the hypotheses made to define it.

\subsubsection{Electron energy distribution deduced from observations}

We thus adopted another strategy in order to estimate the primary electron energy distributions from the observations. As described in Section 4.2.1, the energy flux $E_{f}$ and mean energy $\langle E\rangle$ have been determined from the brightness and CR of each binned pixel of several auroral regions of the STIS observations. Panel a of Fig. 4 clearly demonstrates that the $E_{f}$ and $\langle E\rangle$ are (1) very similar for a given auroral region and (2) very different for different auroral regions. This characteristic can be used as a constraint to empirically determine the energy distribution of the primary electrons of each auroral region. Assuming that the emission at each binned pixel $i$ of a given region is due to electrons in a Maxwellian distribution of mean energy $\langle E(i)\rangle$ and energy flux $E_{f}(i)$, the primary electron distribution that best describes on average this region is thus the sum of the $\langle E(i)\rangle$ Maxwellians, weighted by the $E_{f}(i)$.

The average primary electron energy distributions obtained from this procedure are shown in Fig. 6 for ME 1, ME 2, and FL regions for the two STIS observations. Distributions are not provided for the DC (because of very weak brightness, $\langle E\rangle$ cannot be safely determined for each pixel), IFP (because absorption when present, is at the limit of detection, which does not permit an accurate estimate of $\langle E\rangle$ ), and GFP and SSS (because of the low number of points and large dispersion in the distributions) regions.

To provide an analytical form of these constrained distributions, the latter have been fitted with Maxwellian, kappa and generalized Maxwellian distributions as described by Eqs. (1)-(3). As seen in Fig. 6, each auroral region in both STIS observations has very similar electron energy distribution and thus shares specific characteristics, as already mentioned. At the exception of the ME 1 region whose constrained electron distribution can be well fitted by a Maxwellian distribution, kappa distributions with a spectral index between 3 and 9 usually provide better fits to the ME 2 and FL constrained distributions than Maxwellians do. Indeed, the high energy 'tail' observed in these constrained distributions is better reproduced by the slower decrease of the kappas with energy, 

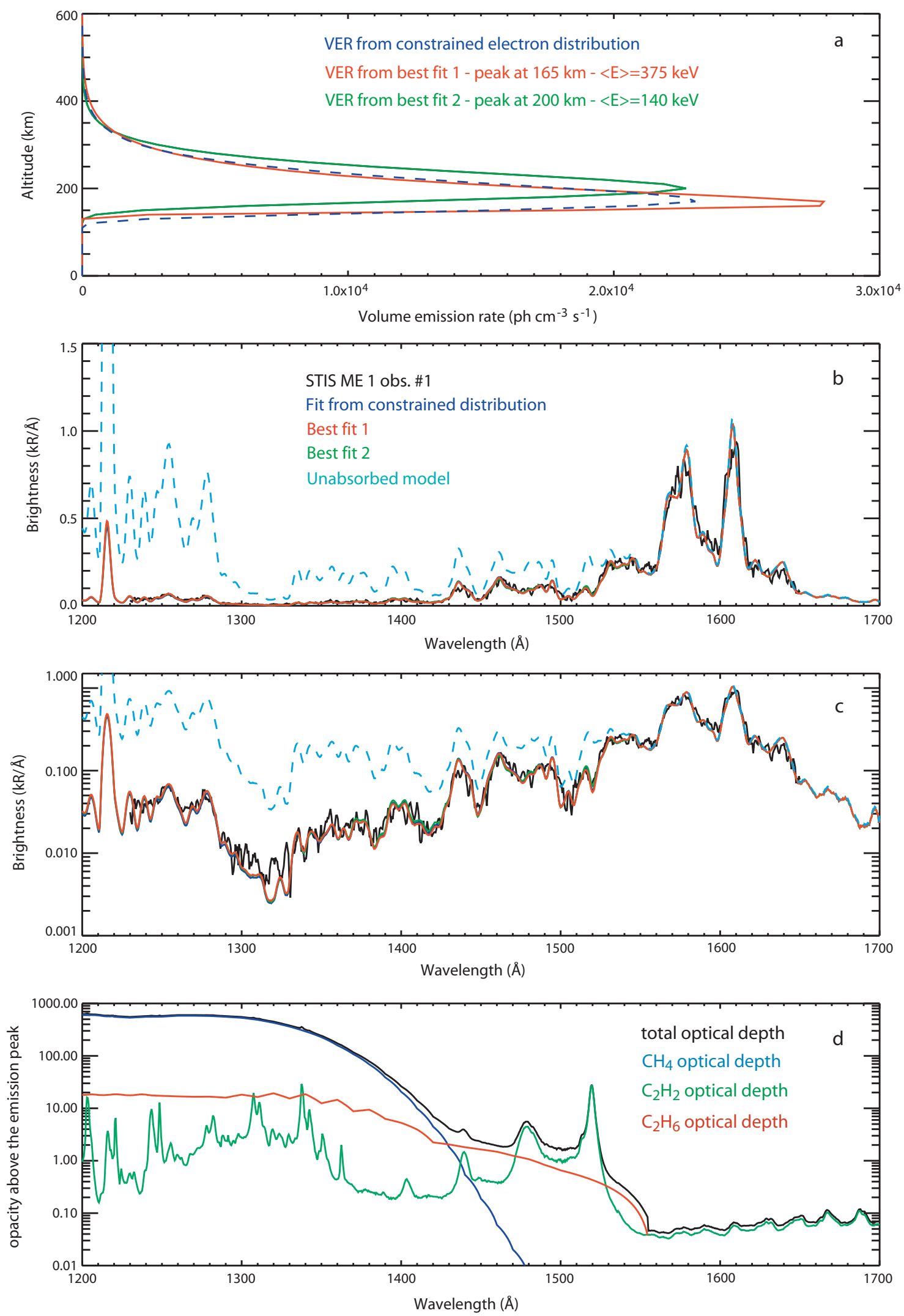

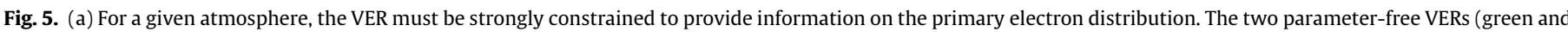

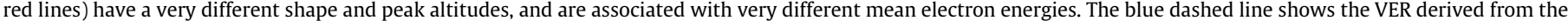

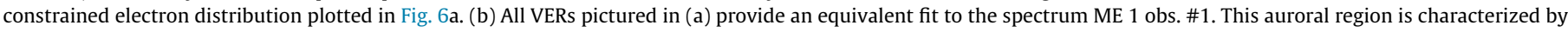

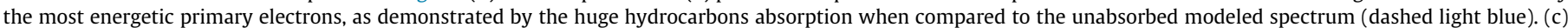

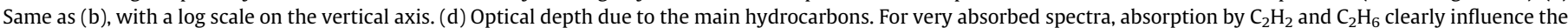

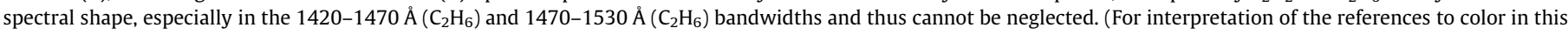
figure legend, the reader is referred to the web version of this article.) 

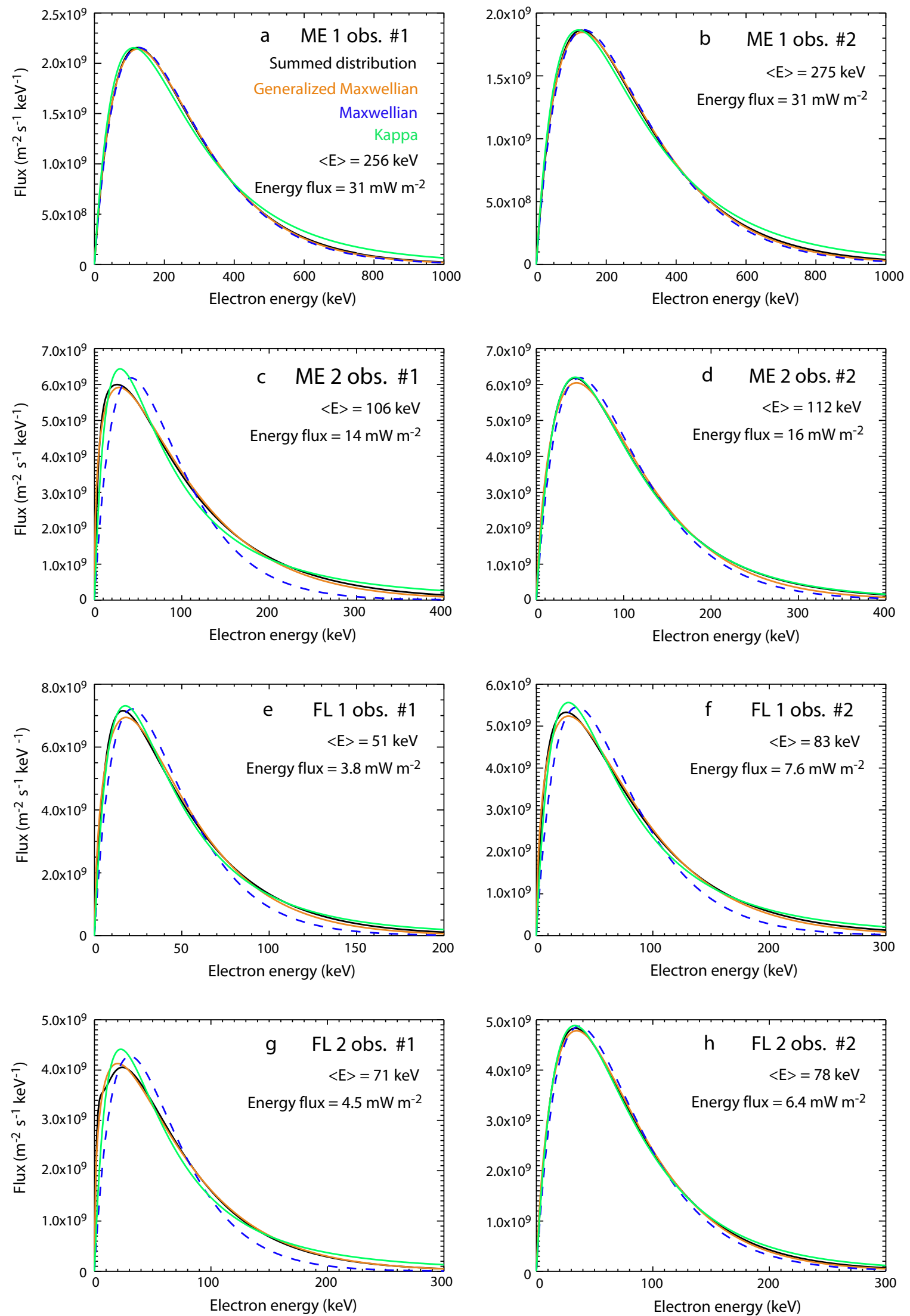

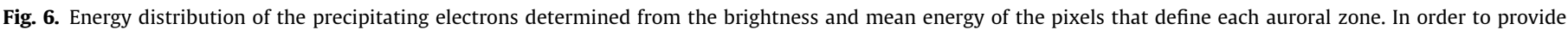

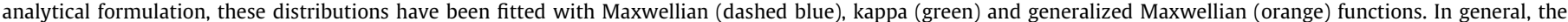

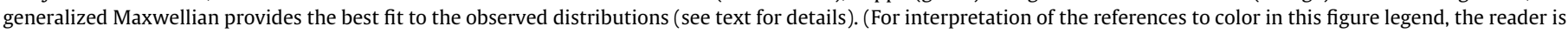
referred to the web version of this article.) 
Table 2

Fit parameters of the electron distributions inferred from the binned pixels.

\begin{tabular}{|c|c|c|c|c|c|c|}
\hline & \multicolumn{5}{|c|}{ Generalized Maxwellian } & \multirow{2}{*}{$\begin{array}{l}\text { Kappa } \\
\text { Spectral index }\end{array}$} \\
\hline & Mean energy (keV) & Energy flux $\left(\mathrm{mW} / \mathrm{m}^{2}\right)$ & Constant factor $C$ & $E_{0}$ & Parameter $n$ & \\
\hline Main emission 1 obs. \#1 & 256 & 30.7 & $7.69 \times 10^{11}$ & 132.4 & 1.87 & 8.04 \\
\hline Main emission 1 obs. \#2 & 275 & 30.9 & $7.31 \times 10^{11}$ & 146.2 & 1.80 & 7.96 \\
\hline Main emission 2 obs. \#1 & 106 & 13.9 & $9.06 \times 10^{11}$ & 71.3 & 0.79 & 3.17 \\
\hline Main emission 2 obs. \#2 & 112 & 16.0 & $9.38 \times 10^{11}$ & 58.5 & 1.57 & 8.75 \\
\hline Flare 1 obs. \#1 & 51 & 3.8 & $5.13 \times 10^{11}$ & 29.7 & 1.21 & 5.10 \\
\hline Flare 1 obs. \#2 & 83 & 7.6 & $6.38 \times 10^{11}$ & 50.8 & 1.09 & 4.48 \\
\hline Flare 2 obs. \#1 & 71 & 4.5 & $4.50 \times 10^{11}$ & 51.1 & 0.78 & 3.81 \\
\hline Flare 2 obs. \#2 & 78 & 6.4 & $5.38 \times 10^{11}$ & 42.0 & 1.64 & 9.16 \\
\hline
\end{tabular}

compared to the Maxwellians. Overall, the generalized Maxwellian functions provide the best fit in each case. The main characteristics of these distributions are provided in Table 2. As expected, the mean energy of the distributions is close to the mean energy determined from the spectra with the CR method shown in Table 1. The VER associated with the constrained distribution corresponding to region ME 1 obs. \#1 (shown in dashed dark blue in Fig. 5a) has been used to generate an emergent synthetic $\mathrm{H}_{2}$ spectrum. This modeled spectrum compares very well to the observed spectrum. In particular, all the spectral features influenced by the $\mathrm{CH}_{4}, \mathrm{C}_{2} \mathrm{H}_{2}$ and $\mathrm{C}_{2} \mathrm{H}_{6}$ absorptions are nicely reproduced by the model, which validates the method performed to derive these constrained primary electron distributions and shows that the $\mathrm{CH}_{4}, \mathrm{C}_{2} \mathrm{H}_{2}$ and $\mathrm{C}_{2} \mathrm{H}_{6}$ density profiles adopted in our model are appropriate to describe the auroral atmosphere.

\subsubsection{The Io footprint: a special case}

In contrast to the main oval and polar cap emissions, a determination of $\langle E\rangle$ from hydrocarbon absorption is difficult for the IFP because of its weak hydrocarbon attenuation. In theory, at a viewing angle of $70^{\circ}$, the CR is 1.2 for $\langle E\rangle=20 \mathrm{keV}$ and 1.5 for $\langle E\rangle=30 \mathrm{keV}$. A $50 \%$ increase of the mean energy thus only changes the $C R$ by 0.3 . This small CR increase is below the limit of accuracy reached by the spectra associated with individual IFP pixels. Deriving an average electron energy distribution is thus not possible from our data. However, several results can be addressed from the analysis of the IFP spectra obtained from the sum of all the IFP pixels.

1. Based on the $C R$ method, the $C R$ of the average spectra suggest an electron mean energy of $\sim 20 \mathrm{keV}$ for both IFP observations. Fig. 7a shows an emission profile (VER 1, orange line) determined from a Chapman profile with free parameters that fits well the IFP spectrum of the 16 Jan. 2013 STIS observation (quasi-identical to the 24 Jan. observation). Its characteristics correspond well to VERs obtained from a Maxwellian electron distribution. It has a narrow shape (FWHM $=125 \mathrm{~km}$ ), peaks at $330 \mathrm{~km}$ and has a CR of 1.24 , compared to 1.28 for the observation. As seen in Fig. 12a, this altitude peak corresponds to a mean energy of $17 \mathrm{keV}$, in agreement with the $C R$ method, accounting for the uncertainties.

2. Apart from the hydrocarbon absorption of the spectra, several other parameters of the IFP emission are constrained by observations. First, vertical emission profiles of the IFP have been determined from ACS images by Bonfond et al. (2009). These authors selected 38 observed VERs from the IFP where the auroral curtain is quasi perpendicular to the observer in order to generate a 'typical' emission profile with good $\mathrm{S} / \mathrm{N}$ ratio. This emission is characterized by a profile peaking at $\sim 900 \mathrm{~km}$ and a FWHM of $\sim 1200 \mathrm{~km}$, much larger than the values attained by VER profiles due to Maxwellian distributions (the maximum FWHM of the VER is $\sim 440 \mathrm{~km}$, obtained from a $2 \mathrm{keV}$
Maxwellian electron distribution, as seen in Fig. 12c). Indeed, this ACS emission was best fitted with a modeled VER profile resulting from a kappa distribution of primary electrons with a spectral index $k=2.3, E_{0}=70 \mathrm{eV}$ and $\langle E\rangle=1.1 \mathrm{keV}$. The VER profile best fitting the ACS observations is plotted in blue in Fig. 7a and is used to constrain the shape of the VER used in our model. Second, examination of the full ACS database reveals that the peak altitude of the IFP emission may be quite variable, with values from $\sim 550$ to $\sim 1300 \mathrm{~km}$, which constrains the range of altitudes allowed for the modeled emission peak. A third constraint arises from the minimum altitude reached by the precipitating electrons. With a Maxwellian distribution of $500 \mathrm{keV}$ electrons, the corresponding VER peaks at $160 \mathrm{~km}$ and does not show substantial emission below $135 \mathrm{~km}$ (Fig. 12a). For mono-energetic electrons of $500 \mathrm{keV}$, the electrons are stopped by a total $\mathrm{H}_{2}$ column of $2.3 \times 10^{22} \mathrm{~cm}^{-2}$, which corresponds to an altitude of $148 \mathrm{~km}$ in the atmospheric model used here. These two cases thus agree to conclude that no significant auroral emission can be produced at altitudes below $\sim 135 \mathrm{~km}$, whatever the primary electron distribution used. The shape of the synthetic VER must then be adapted in order to eliminate all emission below $\sim 135 \mathrm{~km}$. From these constraints, we built a synthetic VER from a Chapman profile, whose shape corresponds to the modeled VER determined by Bonfond et al. (2009) that is allowed to peak in the 500$1300 \mathrm{~km}$ range. The modeled emission profile is forced to decrease at low altitude in order to eliminate all emissions below $135 \mathrm{~km}$. The peak altitude and maximum value of the VER profile are the only free parameters of the model from which synthetic spectra are produced until a synthetic spectrum best fitting the IFP spectrum is obtained. A best emission profile, VER 2, that provides a good fit to the spectrum is shown in green in Fig. 7a. The shape of this VER is significantly thinner than the Bonfond et al. (2009) observations because of the limited emission allowed to emerge at low altitudes, as just explained. The maximum emission arises at an altitude $\sim 300 \mathrm{~km}$, significantly lower than the minimum peak altitude of $\sim 550 \mathrm{~km}$ observed by Bonfond et al. (2009). The peak altitude and shape of VER 2 thus make this solution very unlikely, as they do not meet the constraints provided by the observed emission profiles.

3. Tests reveal that the only way to derive an absorbed spectrum associated with a "thick" VER peaking at an altitude higher than $550 \mathrm{~km}$ is to change the altitude of the homopause level relative to the auroral emission and thus allowing an upwelling/vertical transport of the hydrocarbon layer. In addition to the peak altitude and maximum value of the synthetic VER emission, we thus added a third free parameter in the analysis, i.e. an upward shift of the hydrocarbon density profiles in our atmospheric model. Several fits with different shifts of the hydrocarbon homopause provided comparably good fits to the observed IFP spectrum as shown in Fig. 7c (stars). It is seen that the peak 

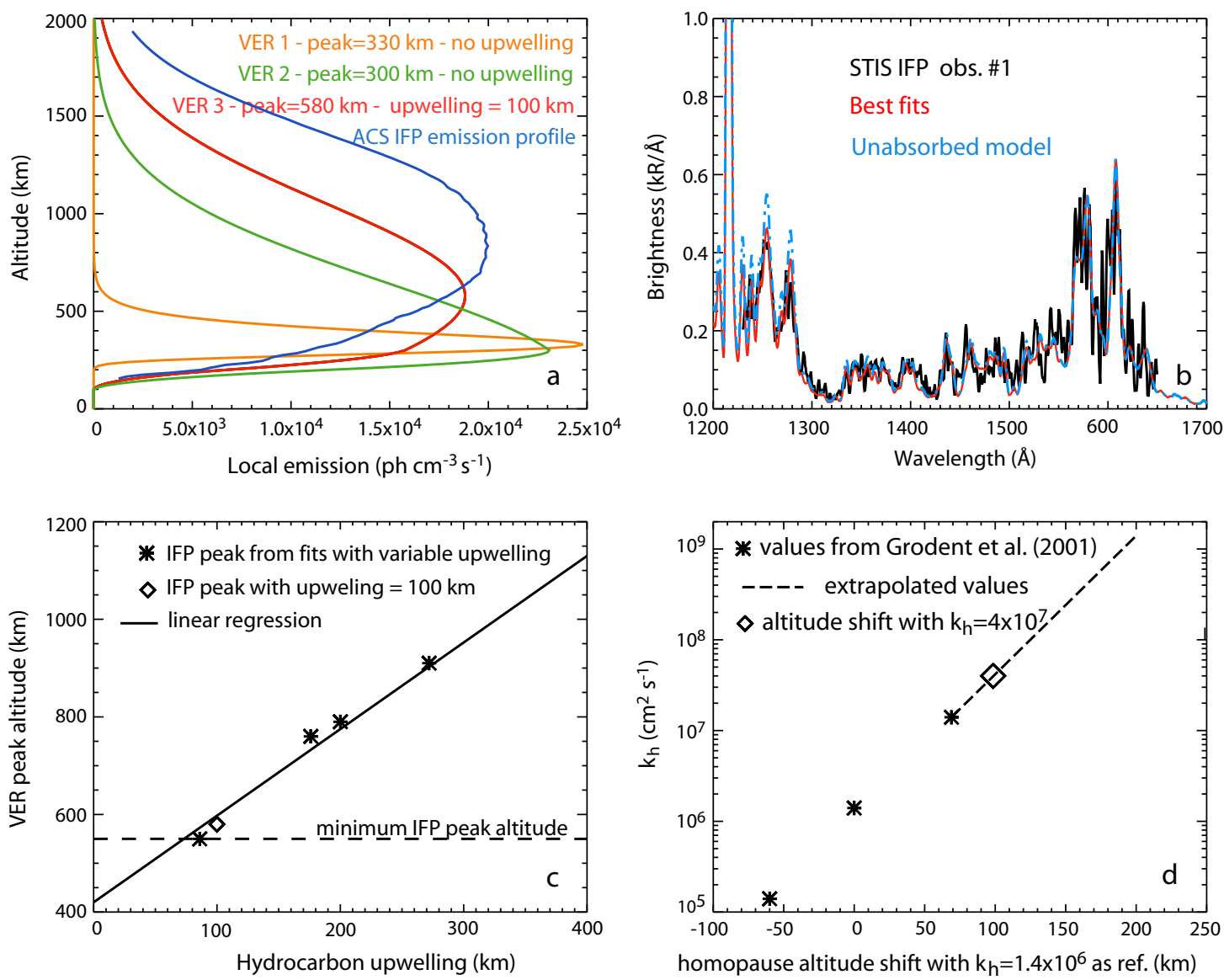

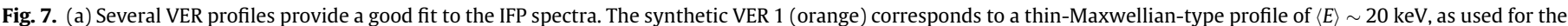

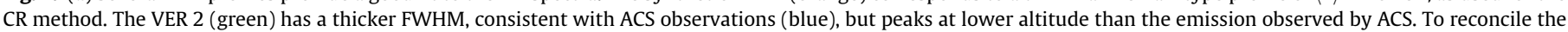

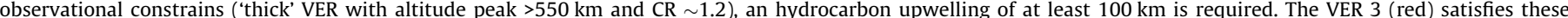

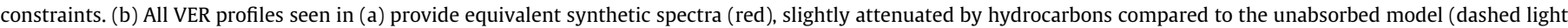

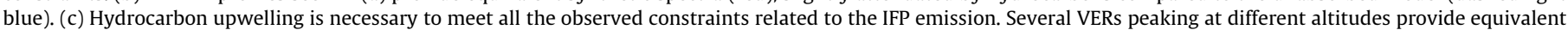

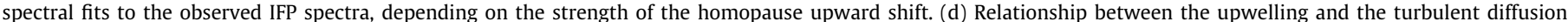

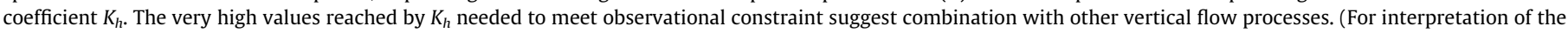
references to color in this figure legend, the reader is referred to the web version of this article.)

altitude of the best VERs varies linearly with the upwelling, which is expected as the absorption depends on the relative position of the VER with the hydrocarbon profiles. Interestingly, a minimum upwelling of $70 \mathrm{~km}$ is required to obtain a VER peaking at $550 \mathrm{~km}$. Assuming that a redistribution of hydrocarbons at higher altitude is exclusively due to a change of the eddy diffusion coefficient $K_{h}$, a $70 \mathrm{~km}$ upward shift of the homopause corresponds to $K_{h}=1.4 \times 10^{7} \mathrm{~cm}^{2} \mathrm{~s}^{-1}$, i.e. 10 times the nominal value used in the Grodent et al. (2001) atmosphere. However, the association of an auroral peak at $550 \mathrm{~km}$ with a CR of 1.3 is very unlikely, as $550 \mathrm{~km}$ is already the lowest altitude observed from the ACS images, and 1.3 is in the lower range of $C R$ values determined so far from IFP spectra (CR from 1.44 to 2.28 where obtained by Gérard et al. (2002) from STIS spectra). Assuming that the relative uplift of hydrocarbon is exclusively due to a variation of $K_{h}$, a maximum homopause shift can be estimated. Results from the Grodent et al. (2001) model show that the homopause altitude varies linearly with $K_{h}$ in log scale (stars in Fig. 7d). On the other hand, Parkinson et al. (2006) analyzed UVIS observation of the He $584 \AA$ emission during the Cassini flyby of Jupiter and determined that $K_{h}$ at the homopause in the auroral region is 'possibly greater' than $4 \times 10^{7} \mathrm{~cm}^{2} \mathrm{~s}^{-1}$. Extrapolation of the relationship presented in Fig. $7 \mathrm{~d}$ to $K_{h}=4 \times 10^{7} \mathrm{~cm}^{2} \mathrm{~s}^{-1}$ leads to a homopause shift of
$100 \mathrm{~km}$. By fixing this value in our fitting procedure, we found a VER best fitting the IFP spectrum peaking at $580 \mathrm{~km}$ (red curve in Fig. 7a and b, diamond in Fig. 7c), which is still in the very low range of altitudes determined by Bonfond et al. (2009).

4. To obtain an IFP emission peaking at a 'standard' altitude of $\sim 900 \mathrm{~km}$ that generates an absorbed emission, an homopause upward shift of $280 \mathrm{~km}$ is necessary. Based on the extrapolation shown in Fig. 7d, this corresponds to an increase of $K_{h}$ to $2 \times 10^{11} \mathrm{~cm}^{2} \mathrm{~s}^{-1}$, i.e. five orders of magnitude above the nominal value in the Grodent et al. (2001) model, which seems very unlikely. However, an increase of turbulent diffusion is not the only way to change the hydrocarbon vertical distribution. Vertical wind transport generated by the auroral precipitation may carry hydrocarbon molecules to altitudes higher than the expected homopause level, thus affecting the absorption of the emergent emission (see discussion in Bonfond et al. (2009)). It should also be noted that the 23 spectral observations of the IFP obtained so far (see Dols et al. (2000) and Gérard et al. (2002, 2014) and the present work) all exhibit hydrocarbon absorption but may all correspond to the lower range of altitudes obtained by Bonfond et al. (2009). Since the ACS images examined by Bonfond et al. (2009) do not provide information on the hydrocarbon absorption, such hypothesis cannot be excluded. 

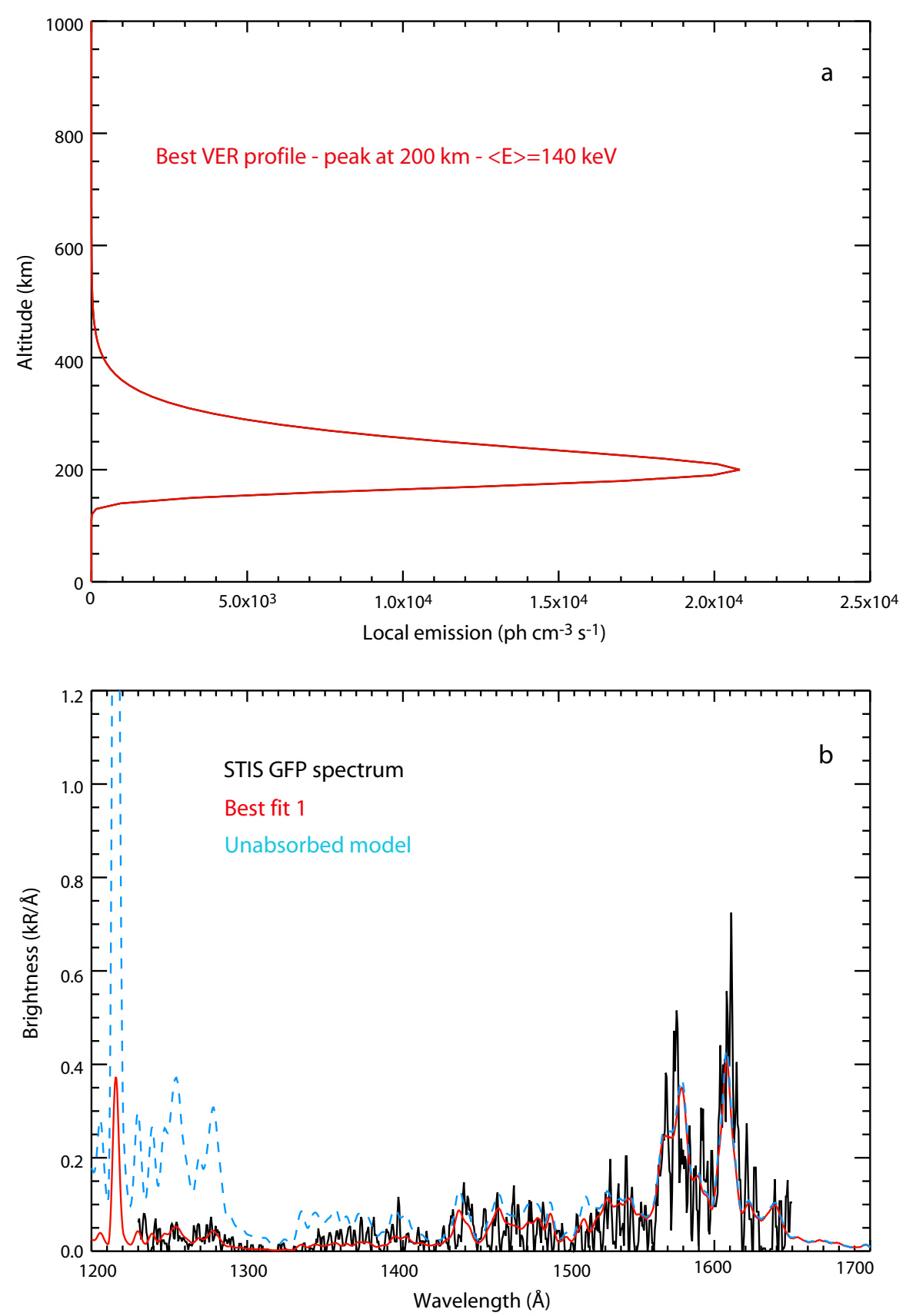

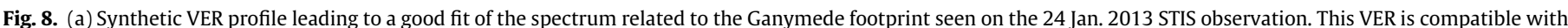

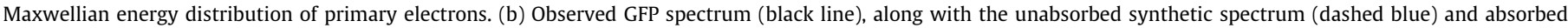

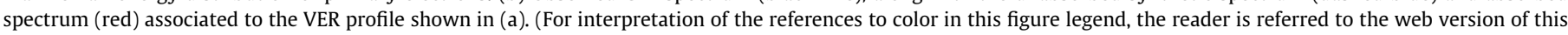
article.)

5. It is quite obvious that the emission profiles presented in Fig. 7a cannot result from Maxwellian electron distributions. Except for the first case discussed (VER 1 compatible with a Maxwellian distribution, orange curve in Fig. 7a), the other emission profiles show a much larger scale height. Thanks to the two free parameters of the kappa distribution, both the shape and altitude of the emission profile can be adjusted by varying $E_{0}$ and the spectral index $k$. Since several (VER profile, homopause shift) configurations simulate well the IFP emission in the framework of the broad constraints just discussed, the electron characteristics determined by Bonfond et al. (2009) cannot be updated here. Still, as a rule of thumb, one can consider that an increase of $E_{0}$ with constant $k$ in the electron energy distribution will favor emission at lower altitude stemming from a shift of the electron energy toward larger values, and thus shift the whole emission profile toward lower altitudes. Keeping $E_{0}$ constant and decreasing $k$ (i.e. increasing the contribution of high energy electrons in the 'tail' of the distribution) drastically changes the shape of the distribution. The low energy electrons conserved by keeping the value of $E_{0}$ constant will keep producing significant emission at high altitudes, while the energetic electrons in the tail of the distribution will increase emissions at low altitude and provide a profile with a shape like VER 2 in Fig. 7a. 
In summary:

(1) With the hydrocarbon distribution in the nominal atmospheric model of Grodent et al. (2001), no emission profile can provide at the same time an absorbed emergent spectrum (as constrained by STIS spectra) and VER characteristics consistent with the ACS constraints.

(2) The only way to reconcile the constraints of absorption and the observed profiles is to significantly shift upward the altitude of the homopause level, by at least $100 \mathrm{~km}$.

(3) The CR method used so far to determine $\langle E\rangle$ is not appropriate for the IFP emission, because (1) the FWHM of the VER profiles obtained from monokinetic or Maxwellian electron distribution is not consistent with the much 'thicker' observed profiles of the IFP emission, and (2) the altitude of the emission peak obtained from the CR method are significantly lower than the range of values deduced from ACS observations. Consequently, $\langle E\rangle$ determined by the CR method (40-70 keV in Gérard et al. (2002) and up to $100 \mathrm{keV}$ in Gérard et al. (2014) are probably overestimated by, at least, an order of magnitude.

(4) Simulations have been performed to determine the $\langle E\rangle-\mathrm{CR}$ relationship with the procedure and atmosphere described in Appendix A.1, assuming a $100 \mathrm{~km}$ upward shift of the homopause level. With such a modified atmosphere, mean energies are, roughly, lowered by a factor of 3 for a given CR.

(5) Processes that could lead to such an important upward shift of the hydrocarbon layer with respect to the auroral emission are not well understood. Contrary to the main emission, which is the result of precipitating electrons accelerated by a quasi-static potential, acceleration of electrons by Alfven waves are involved in the IFP emission (Bonfond et al., 2008; Hess et al., 2010). This difference could be a clue implying that this uplift of the hydrocarbon layer applies to the IFP region only. In terms of the characteristic of the precipitating electrons, the main difference between the Io and main oval or flare emissions is the current density. The energy input is very similar in both cases, but the mean energy of the electrons lies in the range $50-270 \mathrm{keV}$ for the polar emissions and around $1 \mathrm{keV}$ for the IFP emission. This difference directly induces a current density that is $\sim 20$ to $\sim 250$ times higher in the case of the IFP. For example, an energy input of $10 \mathrm{~mW} \mathrm{~m}^{-2}$ leads to a current density of $0.1 \mu \mathrm{A} \mathrm{m}^{-2}$ for a beam of $100 \mathrm{keV}$ electrons and $10 \mu \mathrm{A} \mathrm{m}^{-2}$ for a beam of $1 \mathrm{keV}$ electrons.

\subsubsection{Small scale structure and Ganymede footprint}

As for the discontinuity and IFP, a distribution of primary electrons based on the data (Section 4.3.3) has not been calculated for the small scale structure emission seen in the Jan. 16 STIS observation and the Ganymede footprint seen in the Jan. 24 observation, because of the low number of pixels involved and thus low statistics. Interestingly, the extracted spectra show very important hydrocarbon attenuation for both emissions, with a CR of 11 and 6.7 for the SSS and the GFP, respectively. By using the CR method, these values correspond to $\langle E\rangle$ of 232 and $157 \mathrm{keV}$, respectively. Since the primary electron distribution and the shape of the emission profile are unknown for these emissions, the spectral analysis is identical in both cases. We present in Fig. 8 the analysis of the GFP emission, first tested with a 'thin' emission profile to fit the observed spectrum. A best fit of the GFP spectrum associated with a thin Maxwellian-type VER profile is presented in Fig. 8a and b. The emission peaks at $200 \mathrm{~km}$, which corresponds to energies of $140 \mathrm{keV}$, relatively close to the $157 \mathrm{keV}$ value obtained from the $\mathrm{CR}$ method. The difference is explained by the fact that our regression method tries to minimize differences between the modeled and observed spectrum over the full 1250-1700 ̊ spectral region and not only the bandwidths associated with the CR. Several spectral features of the GFP spectrum are not well defined because of the low $\mathrm{S} / \mathrm{N}$, due to the low number of pixels used to define this region. Still, comparison with an unabsorbed $\mathrm{H}_{2}$ spectrum (dashed blue line in Fig. 8b) clearly demonstrates that the GFP emission is strongly affected by hydrocarbon absorption.

We also tested 'thick' synthetic emission profiles to fit the GFP spectrum, with a method similar to the one used for the IFP in the previous paragraph. It is found that 'thick' emission profiles could not be sufficiently absorbed to provide the observed high CR. If the VER is peaking at altitudes higher than $400 \mathrm{~km}$, the attenuation is too weak to provide high CR, and if the peak is situated at lower altitudes, the shape of the original thick VER changes (decrease of the FWHM) as the emission below $135 \mathrm{~km}$ is strongly dampened in our procedure, in order to account for the altitude limit reached by electrons whatever their energy. Since the vertical thickness of the emission decreases as the emission moves to lower altitude, the shape of the VER becomes similar to the 'thin-Maxwellian type VER', as used in the CR method.

\subsection{Alternative interpretation of UV emission: ion precipitation}

Strong X-ray emissions have been observed over the past 20 years from the polar caps of Jupiter (e.g. Branduardi-Raymont et al., 2008; Ozak et al., 2013 and references therein), with a power on the order of $1 \mathrm{GW}$ for each hemisphere (Elsner et al., 2005). This emission is thought to result from precipitation of oxygen and sulfur ions of several MeV. A model describing the ion precipitation process has been developed by Ozak et al. (2010, 2013). Outputs from this model include secondary electron production rates, field-aligned currents, and auroral emission rates in the UV bandwidth resulting from secondary electrons produced by ion precipitation. Interestingly, this model predicts an unabsorbed emission of 60-80 kR in the north and 10-20 kR in the south in the Lyman and Werner bands of $\mathrm{H}_{2}$, assuming a total X-ray emitted power of $1 \mathrm{GW}$. This corresponds to a 'UV efficiency' of $\sim 3 \mathrm{kR}$ per incident $\mathrm{mW} \mathrm{m}^{-2}$ from ion precipitation, i.e. on average a third of the electron precipitation efficiency. These authors tested precipitation of ions with energies 1, 1.5 and $2 \mathrm{MeV} / \mathrm{u}$, the latter considered as an upper limit of plausible values. The vertical emergent UV brightness of $60-80 \mathrm{kR}$ produced by ion precipitation in the northern polar cap corresponds well to the vertical brightnesses obtained from the FL 1 and FL 2 STIS north observations studied here (mean vertical value from 48 to $88 \mathrm{kR}$, Table 1). In addition to the emergent brightness, the UV vertical profiles resulting from ion precipitation can be used in our model to derive the CR associated with ion precipitation, which can be compared with the STIS observations.

The jovian atmosphere adopted by Ozak et al. (2013), first introduced by Maurellis and Cravens (2001), is based on Galileo probe data (Seiff et al., 1996) and remote observations (Sada et al., 1998). In particular, the temperature profile (hence the $\mathrm{H}_{2}$ density profile) used by Ozak et al. (2013) differs from the Grodent et al. (2001) profiles used in the present study. The ion precipitation model by Ozak et al. (2013) has thus been performed with the Grodent et al. (2001) atmosphere in order to derive $\mathrm{H}_{2}$ emission profiles directly compatible with the other outputs from the present work.

The UV VER profiles of $\mathrm{H}_{2}$ emission so obtained are found to peak between 325 and $290 \mathrm{~km}$ for 1 and $2 \mathrm{MeV} / \mathrm{u}$ ions, respectively. They are used in our auroral model as described in Appendix A. 1 and the related emergent spectra are determined for $\chi=70^{\circ}$, i.e. the typical viewing angle corresponding to the 'flare' emissions seen in our observations. It is seen that the CR due to the UV emission resulting from ion precipitation varies from 1.10 to 1.34 for $1 \mathrm{MeV} / \mathrm{u}$ to $2 \mathrm{MeV} / \mathrm{u}$ ions, respectively (black curves in Fig. 11b). 
These values are significantly lower than the CR from 2.0 to 3.2 obtained from the 'flare' emissions observed in the present study. The latter values correspond well to previous determinations of the CR obtained so far. Fifteen polar cap spectra observed by STIS have been examined by Gustin et al. (2004a). They were characterized by $C R$ values from 1.7 to 7.5 : one $C R$ at 1.7 , five CRs between 2.1 and 3, and nine CRs above 3. Several observations from this data set were obtained in the time tag mode, allowing inspection of transient events (Gérard et al., 2003). These data reveal that only two flaring events were associated with a CR of 1.7 , nine with a CR between 2 and 3 and two with a CR higher than 3. These two studies thus reveal that $89 \%$ of the observed flare emissions (93\% for Gustin et al. (2004a,b) and 85\% for Gérard et al. (2003)) are associated with CRs higher than 2, leaving $\sim 10 \%$ of the cases with a CR of 1.7. Referring to these observed CRs compared to the CR simulated from the Ozak et al. (2013) outputs, polar UV emission exclusively due to ion precipitation is thus very unlikely. Still, a partial contribution of ions to the total UV emission is possible. The effect of the ion precipitation, combined with electron precipitation, would be to lower the observed CR and thus underestimate the mean energy of the precipitating electrons. The $60-80 \mathrm{kR}$ UV brightnesses resulting from this modeled ion precipitation are relatively low and correspond to 'typical' polar cap emissions. Much brighter emissions have been observed at high latitudes. For example, a spectacular event has been observed simultaneously by Chandra in X-ray and HST/STIS in FUV, on 26 February 2003 (Elsner et al., 2005). During this event, the FUV brightness reached $\sim 5 \mathrm{MR}$ and the emitted X-ray power attained $8 \mathrm{GW}$ and both emissions were somewhat correlated. In this exceptional event, a significant contribution from ion precipitation to the UV emission is possible, as these observed characteristics were not considered by Ozak et al. (2013).

\section{Summary - conclusions}

Several important results have been obtained from this study, both from a theoretical and observational point of view.

1. On the theoretical side, we set up a model of auroral $\mathrm{H}_{2}$ emission in the 700-1700 ̊ bandwidth. This model adopts the auroral atmosphere described in by Grodent et al. (2001). Several Maxwellian distributions of primary electrons at mean energies from 2 to $1000 \mathrm{keV}$ have been used to determine the influence of the energy input on the characteristics of the auroral emissions. Results show that:

- the altitude of the emission peak monotonically decreases as $\langle E\rangle$ increases. Assuming that $1 \mathrm{MeV}$ corresponds to the upper limit possibly reached by primary electrons, virtually no auroral photons can be emitted at altitudes below $\sim 130 \mathrm{~km}$, which corresponds to an $\mathrm{H}_{2}$ column of $\sim 5.5 \times 10^{22} \mathrm{~cm}^{-2}$,

- the efficiency of the atmosphere to produce UV aurora strongly depends on the energy of the precipitating electrons. For a constant energy flux input of $1 \mathrm{~mW} \mathrm{~m}^{-2}$, the brightness in the Lyman and Werner bands of $\mathrm{H}_{2}$ is maximum at $\langle E\rangle=20 \mathrm{keV}(14.6 \mathrm{kR})$ and then continuously decreases for higher energies to reach $3.2 \mathrm{kR}$ at $500 \mathrm{keV}$,

- in an altitude scale, the FWHM of the emission profile due to Maxwellian distributions decreases with increasing mean energy with values from $440 \mathrm{~km}(2 \mathrm{keV})$ to $53 \mathrm{~km}$ (500 keV).

The observed color ratio (CR) has been related to the mean energy of the precipitating electrons $\langle E\rangle$ with a model that includes effects of the emission angle $\chi$ and the width $D$ of the auroral curtain. It is found that
- for a given $\langle E\rangle$, the perceived CR increases with $\chi$,

- for a given $\chi$, the auroral width $D$ has a minor effect on the CR,

- the unabsorbed (intrinsic) brightness strongly depends on $\chi$ and the auroral width considered (Fig. 11).

Our model has been applied to two sets of STIS FUV spectral images of the north jovian aurora. Several auroral regions have been selected in both images: morning main emission 1 (ME 1), main emission 2 (kink region, ME 2), discontinuity (DC) flare emissions (FL 1 and FL 2), Io footprint (IFP), Ganymede footprint (GFP) and small scale structure emission (SSS). Interestingly each region exhibit very specific characteristics, as described hereafter.

\section{Mean energy}

1. The maps of the electron mean energy show that the spatial distribution of the precipitating electron energy is clearly not uniform (Figs. 2 and 3). Because of low $\mathrm{S} / \mathrm{N}$ of several individual pixels, such maps only provide a first order estimate of the mean energy of the precipitating electrons.

2. The spectra obtained from the sum of the pixels defining each auroral region do not bring direct information on the electron energy distribution, but provide the most accurate average mean energy for each region. The morning ME 1 region is the most energetic $(\sim 260 \mathrm{keV})$, followed by the 'kink' ME 2 region $(\sim 110 \mathrm{keV})$ and the polar cap flare emissions $(\sim 75 \mathrm{keV})$. The higher $\mathrm{S} / \mathrm{N}$ of the summed spectra allows determining mean energies for the DC, IFP, GFP and SSS regions, defined from low number of pixels and/or weak signal. Mean electron energies are $\sim 60 \mathrm{keV}$ for the DC, $\sim 155 \mathrm{keV}$ for the GFP, and $\sim 230 \mathrm{keV}$ for the SSS emissions. The CR method, providing energies around $20 \mathrm{keV}$ for the IFP, was found unappropriate for this emission (see Section 4.3.4). The spatial distribution of mean energies corresponds well with the energy map from the 8 Jan. 2014 STIS observation presented in Gérard et al. (2014), which was obtained with comparable CML, although our $\langle E\rangle$ values are lower.

\section{Mean energy-energy flux relationship}

The pixels in each auroral region have been sorted by ascending brightness and binned by groups of height data points to increase the $\mathrm{S} / \mathrm{N}$ ratio and determine reliable mean energy and energy flux values. The electron energies of the main emission and flare regions are positively correlated with the energy fluxes and follow well Knight's (1973) theory of field-aligned electric field acceleration associated with field-aligned currents. Magnetospheric electron sources have densities around $2400 \mathrm{~m}^{-3}$ for ME 2 with a thermal energy of $2.5 \mathrm{keV}$, which is consistent with a density between $1000 \mathrm{~m}^{-3}$ and $10,000 \mathrm{~m}^{-3}$ measured by Voyager in the jovian magnetospheric equatorial plane (Scudder et al., 1981). The relativistic generalization of Knight's theory developed by Cowley (2006) has been used for ME 1, as the electron's acceleration is a significant fraction of the electron rest energy for this region. Lower electron source densities are obtained (520$620 \mathrm{~m}^{-3}$ ) and correspond well with values obtained from the study of a remarkably bright auroral morning arc observed by STIS on 21 September 1999 (Gustin et al., 2006).

\section{Spectral analysis}

Strong hypotheses on the atmospheric structure (hydrocarbon density profiles, temperature profile), and on the energy distribution of the primary electrons must be postulated to infer the CR$\langle E\rangle$ relationship. The spectral resolution of the STIS G140L observations examined here is too low $\left(>1 \AA\right.$ ) to resolve the $\mathrm{H}_{2}$ rotational lines, which hence does not allow us to derive information on 
the temperature profile of the auroral atmosphere. Still, the $500 \AA$ bandwidth STIS FUV spectra at 12 Å resolution provide more information than the $C R$, which is limited to the ratio of two integrated 70 A bands. We tested the hypothesis that comparisons between synthetic and observed spectra provide information on the energy distribution of the precipitating electrons and on the appropriateness of the atmospheric model used. We use Chapman functions defined with 4 free parameters to simulate the VER profile and tested the hypothesis that a specific VER profile (thus a unique electron energy distribution) provides spectral fits significantly better than any other VER, which would bring additional constraints on primary electron characteristics. It is found that without constraints, several significantly different VER profiles provide equivalent fits to the observed spectra, which does not allow us to provide additional knowledge on the primary electron distributions. Still, simulations show that $\mathrm{C}_{2} \mathrm{H}_{6}$ is required to fit strongly absorbed spectra and that $\mathrm{CH}_{4}, \mathrm{C}_{2} \mathrm{H}_{2}$ and $\mathrm{C}_{2} \mathrm{H}_{6}$ mixing ratio profiles of the model are appropriate to fit the different bandpasses influenced by these hydrocarbons.

In general, the peak of the intrinsic VER emission is situated at lower altitude than the observed peak emission because of the attenuation at and below the hydrocarbon layer. For example, for a CR of 3.2, the difference of perceived altitude is $\sim 45 \mathrm{~km}$.

Since the energy-flux relationship is very specific to each auroral region, we used this property to determine energy distributions of the primary electrons, constrained by the $\langle E\rangle$ and $E_{f}$ values associated to each pixel of a given region (Section 4.3.3 and Fig. 6). It is seen that distributions for ME 1 are Maxwellian, distributions for the ME 2 and flare regions exhibit more electrons at high energy (compared to a Maxwellian distribution). These electron distributions provide ad hoc solutions able to represent the average emission in each region.

Unlike the other regions, ACS images provide observed constraints on altitude and shape of the VER profile of the IFP emission (Bonfond et al., 2009). The spectral analysis shows that a synthetic VER profile consistent with a Maxwellian electron energy distribution and the CR method provides a good fit to the IFP spectra, but is incompatible with ACS constraints. Indeed, the Maxwellian-type VER profile is much thinner $(\sim 150 \mathrm{~km})$ and peaks at much lower altitude $(\sim 330 \mathrm{~km})$ than the FWHM of $\sim 1200 \mathrm{~km}$ and peak altitude between 550 and $1300 \mathrm{~km}$ provided by the limb analysis of the ACS images. The CR method is thus not applicable to the IFP emission. Several tests reveal that the only way to reconcile constraints on the observed VER profiles and hydrocarbon absorption altogether is to allow an upward shift of the hydrocarbon layer in our atmospheric model. Such shift would in turn modify the temperature and $\mathrm{H}_{2}$ density profiles of the atmosphere we use, but since these two parameters are not directly constrained by the IFP data, consequences on the atmospheric structure are out of the scope of the present study. Several fits with varying levels of upwelling show that the homopause altitude shift and the peak altitude of the best modeled VER profile are linearly correlated. A minimum upwelling of $100 \mathrm{~km}$ is needed to satisfy all the constraints, but a homopause upward shift of $280 \mathrm{~km}$ is needed to be consistent with an IFP emission peaking at a typical altitude of $\sim 900 \mathrm{~km}$. This corresponds to an increase of $K_{h}$ to $2 \times 10^{11} \mathrm{~cm}^{2} \mathrm{~s}^{-1}$, i.e. 6 orders of magnitude above the nominal value used in the Grodent et al. (2001) model, which seems very unlikely. Other phenomena like vertical winds could account for such hydrocarbon upward shift, but our present data cannot strongly constrain the cause of such a shift.

If the CR method is not appropriate to determine $\langle E\rangle$ in the case of the IFP, this method is found compatible with theoretical models and observed constraints in the case of the other auroral regions. In particular, the SSS and GFP spectra exhibit strong hydrocarbon absorption, which cannot be modeled with 'thick IFP-type' emission profile, but which is compatible with 'thin Maxwellian-type' emission profile, as used in the CR method. It should be kept in mind that the $\mathrm{CR}-\langle E\rangle$ relationship is strongly model-dependent. It is influenced by (1) the temperature profile, (2) the altitude of the hydrocarbon homopause level and (3) the energy distribution of the primary electrons used in the model.

\section{Ion precipitation}

The auroral model described in this paper has been applied to FUV $\mathrm{H}_{2}$ emission profiles resulting from the precipitation of heavy ions in the 1-2 MeV/u range (Ozak et al., 2013), in order to determine if such precipitation could explain, at least partly, polar cap auroral emissions. Results show that the CR of the UV emission due to ions $(<1.4)$ is lower than the CR observed poleward of the main oval $(\geqslant 2)$. A significant contribution of ion precipitation to flare emissions typically observed (brightness $<100 \mathrm{kR}$ ) is thus very unlikely. In case of brighter flare emission, such as the $5 \mathrm{MR}$ of FUV emission recorded by STIS on 26 February 2003 concurrently with Chandra X-ray observation (Elsner et al., 2005), an important contribution of ion precipitation cannot be ruled out.

\section{Acknowledgments}

We thank two anonymous reviewers for their constructive comments and suggestions that allowed us to improve the manuscript. J.G. was supported by the PRODEX program managed by ESA in collaboration with the Belgian Federal Science Policy Office. B.B. acknowledges support from the Belgian Fund for Scientific Research (FNRS). L.C.R. was supported by STFC's UCL Astrophysics Consolidated grant ST/J001511/1. This research is based on observations with the Hubble Space Telescope, obtained at the Space Telescope Science Institute, which is operated by AURA for NASA.

\section{Appendix A. Determination of the mean energy of the primary electrons}

\section{A.1. Auroral model}

Our auroral atmospheric model uses the auroral thermal profile and $\mathrm{H}_{2}, \mathrm{CH}_{4}$ and $\mathrm{C}_{2} \mathrm{H}_{2}$ profiles described in Grodent et al. (2001). It couples a two-stream electron transport model of energy deposition with a 1-D thermal conduction model. From the initial North Equatorial Belt (NEB) atmospheric structure deduced from Galileo observations (Gladstone et al., 1996) adapted to the larger gravitational acceleration near the poles, the Grodent et al. (2001) model self-consistently adapts to the effects of the incident primary electron distribution. At each layer of the atmosphere, the energy distribution of the primary electrons changes as they interact with the atmosphere through ionizations, collisions and creation of secondary electrons. The thermal profile and the $\mathrm{CH}_{4}$ and $\mathrm{C}_{2} \mathrm{H}_{2}$ profiles automatically adapt to the auroral conditions. This model is constrained by observations such as the altitude of the $\mathrm{H}_{2}$ emission peak, the temperature profile deduced from UV and infrared emissions, and UV emission rates deduced from observed images and spectra. All density profiles from this atmosphere, defined from 117 to $3900 \mathrm{~km}$ above the $1 \mathrm{bar}$ level, are used in our model. To extend these profiles to lower altitudes and cover the widest possible altitude range, we merged the density profiles from -65 to $117 \mathrm{~km}$ defined in the low-latitude atmospheric model of Moses et al. (2005), and scaled them to obtain continuous densities in our final auroral atmosphere. Ethane is not included in the Grodent et al. (2001) atmosphere. Since the signature of ethane is clearly observed in the case of strong absorption ( $C R>2$ ), it is included here by using the $\mathrm{C}_{2} \mathrm{H}_{6}$ density profile described by Moses et al. (2005). The vertical density profile in our model is obtained by multiplying the $\mathrm{C}_{2} \mathrm{H}_{6}$ mixing ratio relative to $\mathrm{H}_{2}$ found in Moses et al. (2005) by the $\mathrm{H}_{2}$ density profiles outlined in 

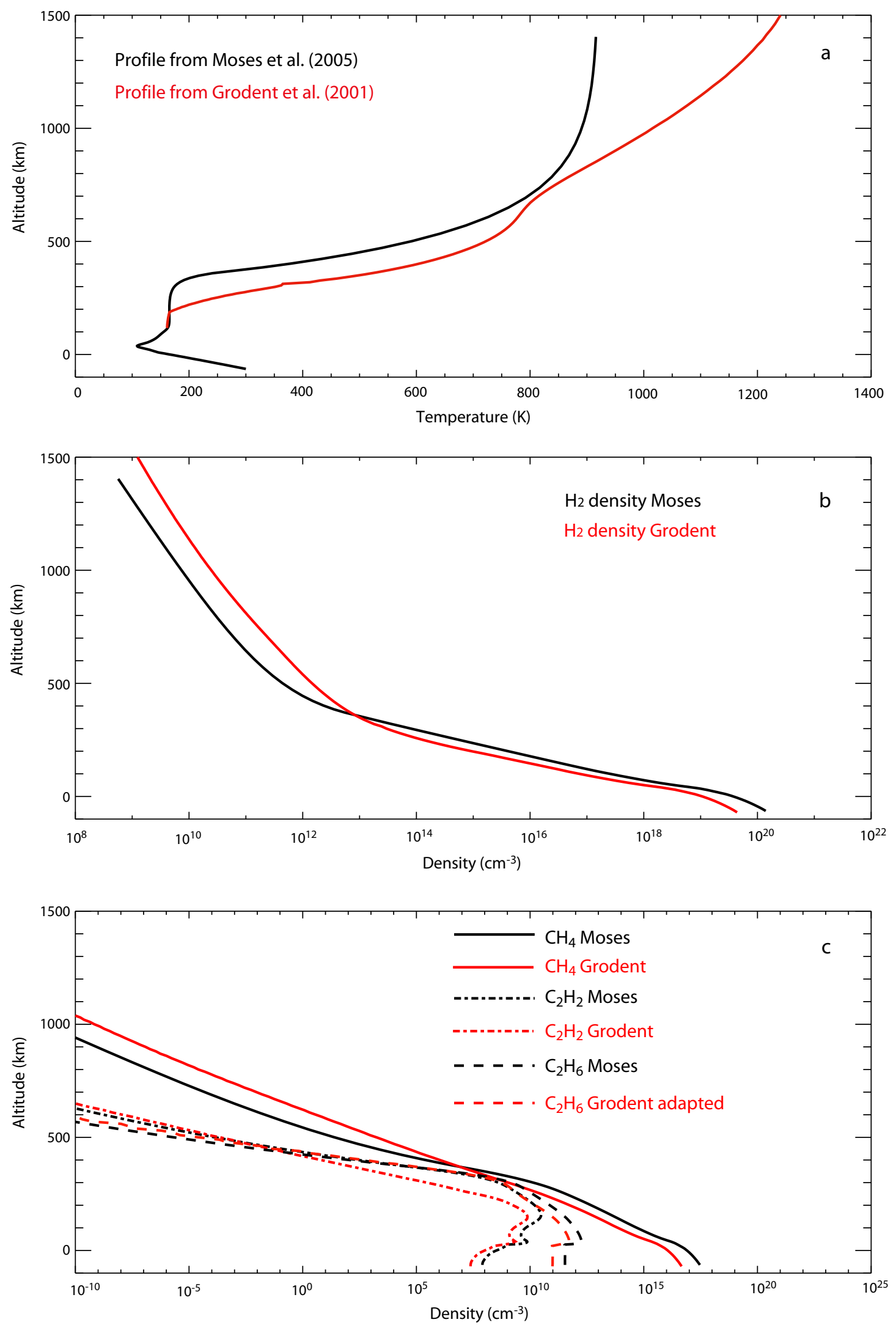

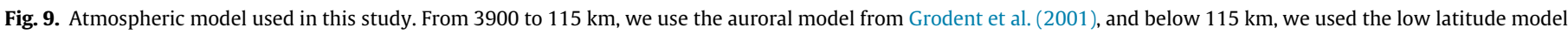

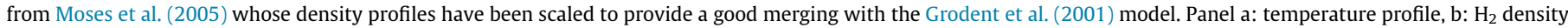
profile, c: density profiles of the main hydrocarbons. 
Grodent et al. (2001). This auroral atmospheric model, along with the Moses model are shown in Fig. 9. The 'hotter' temperature profile of the Grodent et al. (2001) model in Fig. 9a demonstrates the consequence of the electron precipitation on the atmosphere, which also has an effect on the $\mathrm{H}_{2}$ scale height shown in Fig. 9b. The most abundant absorber is methane, followed by ethane and acetylene (Fig. 9c).

To relate $\langle E\rangle$ to $\mathrm{CR}$, we use single Maxwellian distributions with mean energies from 2 to $1000 \mathrm{keV}$, representing the initial energy distribution of the precipitating electrons and covering the widest possible range of mean energies. An auroral volume emission rate vertical profile (VER: number of auroral photons emitted per second per $\mathrm{cm}^{3}$ ) is then produced from each Maxwellian distribution with the two-stream Grodent et al. (2001) model. The atmosphere is divided into altitude bins of $10 \mathrm{~km}$, from -65 to $3900 \mathrm{~km}$ and a normalized unabsorbed synthetic $\mathrm{H}_{2}$ spectrum, weighted by the local VER, is generated at each layer. At altitude bin $z$, the local unabsorbed $\mathrm{H}_{2}$ emission $\mathrm{H}_{2}{ }^{\text {unabs }}(z)$ is

$H_{2}^{\text {unabs }}(z)=H_{2}^{\text {synth }} \times \operatorname{VER}(z) \times d z \times \operatorname{Chap}(z, \chi)$,

where $\mathrm{H}_{2}{ }^{\text {synth }}$ is the unabsorbed $\mathrm{H}_{2}$ spectrum in 700-1700 $\AA$, VER(z) is the auroral emission rate at altitude $z, d z$ is the thickness of a layer and $\operatorname{Chap}(z, \chi)$ is the Chapman function that converts vertical to slant thickness in curved atmosphere (Smith and Smith, 1972). This function substantially differs from the simple $\sec (\chi)$ factor for high $\chi$. We use the gravity and radius at $75^{\circ}$ latitude to compute $\operatorname{Chap}(z, \chi)$, which brings values applicable to the whole polar region.

Accounting for hydrocarbon absorption, the attenuated $\mathrm{H}_{2}$ emission at altitude $z$ is

$H_{2}^{a b s}(z, \lambda)=H_{2}^{u n a b s}(z, \lambda) \times e^{(-\tau(z, \lambda))}$,

where the optical depth $\tau$ at wavelength $\lambda$ at altitude $z$ is

$\tau_{z, \lambda}=\sigma_{\lambda}\left(\mathrm{CH}_{4}\right) \int_{z}^{\infty} n_{\mathrm{CH} 4} d s+\sigma_{\lambda}\left(\mathrm{C}_{2} \mathrm{H}_{2}\right) \int_{z}^{\infty} n_{\mathrm{C} 2 \mathrm{H} 2} d s+\sigma_{\lambda}\left(\mathrm{C}_{2} \mathrm{H}_{6}\right) \int_{z}^{\infty} n_{\mathrm{C} 2 \mathrm{H} 6} d s$,

where $\sigma_{\lambda}\left(\mathrm{CH}_{4}\right), \sigma_{\lambda}\left(\mathrm{C}_{2} \mathrm{H}_{2}\right), \sigma_{\lambda}\left(\mathrm{C}_{2} \mathrm{H}_{6}\right)$ are the methane, acethylene and ethane absorption cross sections at wavelength $\lambda$, and $n_{\mathrm{CH} 4}, n_{\mathrm{C} 2 \mathrm{H} 2}$ and $n_{\mathrm{C} 2 \mathrm{H} 6}$ are the local number densities. The slant path length $d s$ corresponds to the thickness of a layer $d z$ multiplied by the Chapman function $\operatorname{Chap}(z, \chi)$, at emission angle $\chi$.

A point that is often overlooked in auroral study is the finite size of the auroral curtain. To account for this aspect, we assume that the auroral emission has a rectangular section of width $D$ that extends from the lowest to the highest limits of the atmospheric model (Fig. 10). Although the whole altitude range is taken into account for ease of computing, only a limited vertical extension contributes to the emergent emission (represented by the shaded region in Fig. 10). The emergent unabsorbed spectrum $\mathrm{H}_{2}{ }^{\text {unabs_tot }}$ (i.e. the intrinsic auroral emission), in $\mathrm{kR}$, at angle $\chi$ is

$H_{2}^{\text {unabs_tot }}=\sum_{i=0}^{n-1} R_{i} / n e$,

where each emergent beam ray $R_{i}$ is the sum of the local emissions $\mathrm{H}_{2}{ }^{\text {unabs }}$ defined by (6) intercepted by $\mathrm{R}_{i}$ inside the rectangle of width $D$ (dots in Fig. 10), $n$ is the total number of rays considered, and $e$ is the minimum distance between two consecutive rays. Similarly, the emergent $\mathrm{H}_{2}$ spectrum attenuated by the hydrocarbon layer at and above the emission is given by Eq. (9) where $\mathrm{H}_{2}{ }^{\text {unabs }}$ is replaced by $\mathrm{H}_{2}{ }^{a b s}$ defined by Eq. (7). The resulting synthetic emergent spectrum represents the observed, potentially absorbed, emission as seen by an observer, from which a CR can be determined.

It should be emphasized here that the kilo-Rayleigh is a 'brightness density', as it is defined 'per unit of emitted surface'. On the one hand, if one takes into account rays that do not cross substantial emission (i.e. far from the emission peak, e.g. $R_{j}$ in Fig. 10 ), this

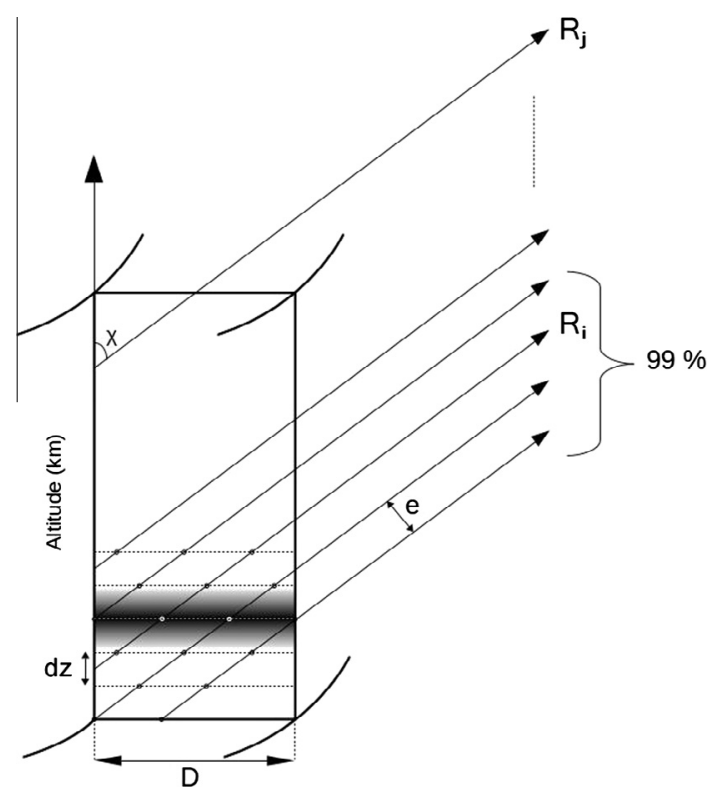

Fig. 10. Inclusion of the width of the auroral curtain ' $D$ ' in the model. Rays 'Ri' emitted at angle $\chi$ and separated by ' $e$ ' $\mathrm{km}$ are summed to represent the total emergent emission. For each $R i$, a limited number of 'emitting points', due to different layers of thickness $d z$, contribute to the emission.

would significantly lower the total brightness of the emergent emission. On the other hand, selection of the brightest rays close to the emission peak alone artificially enhances the perceived emergent brightness of the whole emission. In a nadir view $\left(\chi=0^{\circ}\right)$, the procedure illustrated in Fig. 10 is not necessary, as the emergent brightness is simply the sum of the vertical VER at each layer multiplied by the vertical thickness $d z$ of a layer. It is thus independent of the number of rays considered for an homogeneous emission. We use this vertical emergent brightness to determine the number of $R_{i}$ that provides a smooth transition between vertical and slant emission. We thus determined the emergent unabsorbed $\mathrm{H}_{2}$ intensity and $\mathrm{CR}$ at $\chi=5^{\circ}$ for various number of rays at fixed distance $e$ by varying $n$ in Eq. (9). We found that the number of $R_{i}$ considered in the sum leading to $99 \%$ of the total emission provides smoothest transition of $\mathrm{CR}$ and brightness between $\chi=0^{\circ}$ and $5^{\circ}$. This number varies with the width of the VER profile and the viewing angle considered.

To simulate the effect of the auroral width $D$, the mean energy $\langle E\rangle$ and the viewing angle $\chi$ on the emergent unabsorbed and absorbed $\mathrm{H}_{2}$ emission, we used several single Maxwellian electron distributions from $\langle E\rangle=2$ to $1000 \mathrm{keV}$ and determined the corresponding auroral VER by using the two-stream model from Grodent et al. (2001). We applied these VER in our model for various auroral widths and viewing angles from $0^{\circ}$ to $85^{\circ}$. Several outputs from this model are summarized in Fig. 11. Fig. 11a and b shows the $\mathrm{CR}$ as function of the incident electron energy $\langle E\rangle$, for an auroral curtain of arbitrary width $D=1500 \mathrm{~km}$. It is seen that the effect of the viewing angle is far from negligible: compared to vertical values, the CR increase at $\chi=70^{\circ}$ is less than $5 \%$ for $\langle E\rangle \leqslant 20 \mathrm{keV}, \quad 30 \%$ for $\langle E\rangle=100 \mathrm{keV}$ and reaches $41 \%$ for $\langle E\rangle=400 \mathrm{keV}$.

The effect of the auroral width on the emergent unabsorbed brightness is shown in Fig. 11c for primary electrons of $80 \mathrm{keV}$ (which can be seen as an 'average' energy in the case of the jovian aurora). Four cases are examined: $D=150 \mathrm{~km}$, which is used for footprint emissions, $D=600 \mathrm{~km}$, which represents a narrow auroral emission comparable to the main oval, $D=1500 \mathrm{~km}$ which can represent the thicker, more diffuse emissions, as seen poleward of the main oval, and a case with $D=10,000 \mathrm{~km}$, which 

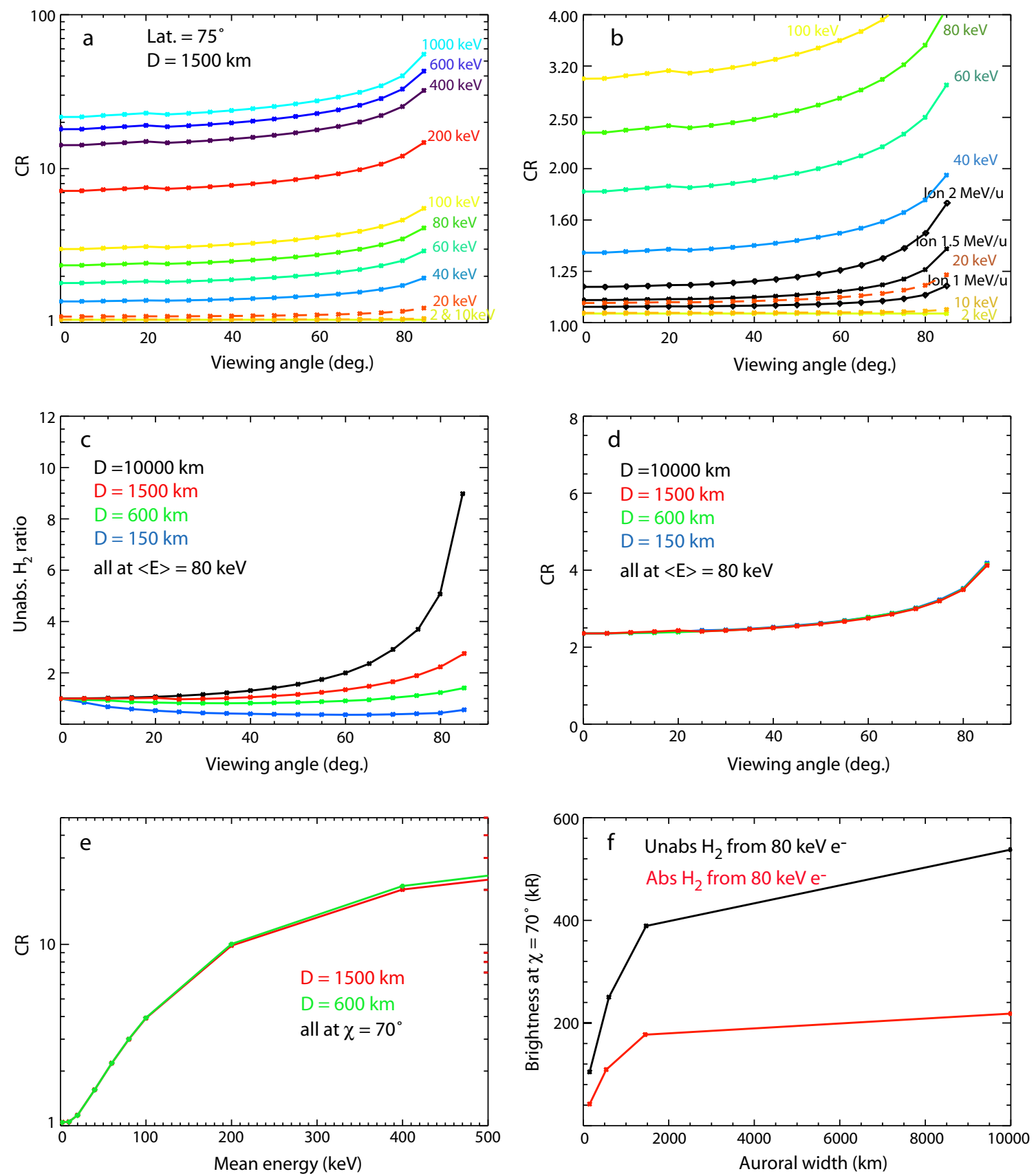

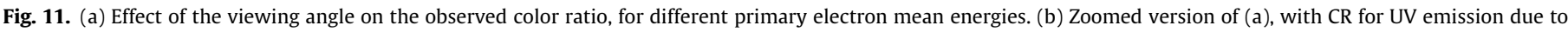

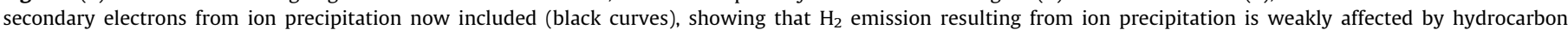

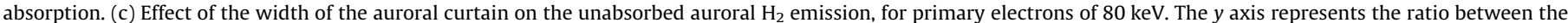

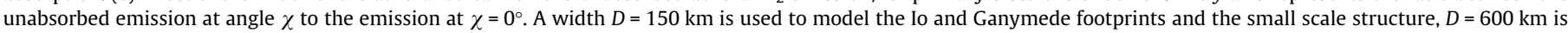

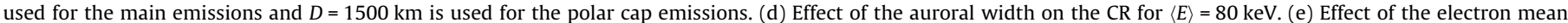

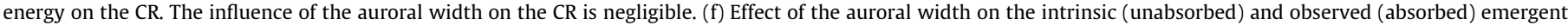

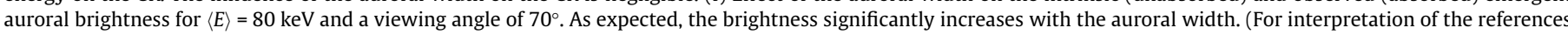
to color in this figure legend, the reader is referred to the web version of this article.)

depicts an homogeneous "infinite" auroral emission. The latter case simulates the effect of the viewing angle on the $\mathrm{H}_{2}$ brightness and $\mathrm{CR}$ when the auroral width is not taken into account in the model. For a viewing angle of $70^{\circ}$, the brightness increase is very important for the infinite case (increase by a factor of 2.8), and then decreases as the aurora is getting thinner (factor of 1.66 , 1.04 and 0.39 for $D=1500,600$ and $150 \mathrm{~km}$, respectively). Interestingly, for $D=150 \mathrm{~km}$, the emergent brightness decreases with the viewing angle. This counter intuitive geometric effect is due to the limited vertical extent of the auroral emission. For example, simulations show that the VER profile obtained from a $80 \mathrm{keV}$ Maxwellian distribution peaks at $226 \mathrm{~km}$ and has a FWHM of $106 \mathrm{~km}$. The portion of the emission centered on $226 \mathrm{~km}$ and of width $150 \mathrm{~km}$ corresponds to $85 \%$ of the total emission. To first order, the transverse cut of an $80 \mathrm{keV}$ auroral curtain of $150 \mathrm{~km}$ width can thus be seen as a square of $150 \times 150 \mathrm{~km}$ width. The observed emission at $\chi=0^{\circ}$ and $90^{\circ}$ should then be very similar, which is what we observe in the blue 
curve of Fig. 11c. For intermediate viewing angles, although the total emitted brightness is unchanged, the total surface perceived by the observer increases with $\operatorname{secant}(\chi)$. Since the kilo-Rayleigh represents an observed brightness per emitting surface unit, the observed brightness decreases for increasing $\chi$. This effect diminishes as the auroral width increases, as it is compensated by the increase of emission width. It should be noted that since the FWHM of the VER profile decreases with increasing $\langle E\rangle$ (see Appendix A.2), the effect of $D$ on the emergent brightness is energy dependent. This characteristic is an important matter and is taken into account in the data analysis. As seen in Fig. 11d, the effect of the auroral width on the CR is quite minor, as the maximum variation due to the auroral width is $3 \%$ at $85^{\circ}$ for $\langle E\rangle=80 \mathrm{keV}$, below the accuracy achieved by the observations. This means that the width affects the absorbed and unabsorbed emissions in a similar way. The $\mathrm{CR}-\langle E\rangle$ relationship is presented in Fig. 11e for a standard viewing angle of $70^{\circ}$. Considering that $70^{\circ}$ is a typical viewing angle of Jupiter's poles as seen from HST, Fig. 11e can be used to estimate the incident precipitating energy from an observed absorbed emission. The effect of the auroral width on the emergent brightness is presented in Fig. 11f, for an incident electron energy flux of $20 \mathrm{~mW} \mathrm{~m}^{-2}$. As expected, the observed brightness increases with the width of the aurora. The point at $10,000 \mathrm{~km}$ on the $x$-axis of Fig. $11 \mathrm{f} \mathrm{sim-}$ ulates the observed brightness that would be obtained without taking into account $D$. The red curve shows the emergent total $\mathrm{H}_{2}$ brightness after attenuation by hydrocarbons. On average, the factor between the unabsorbed and absorbed emission in Fig. $11 \mathrm{f}$ is 2.12 . Another way to interpret this plot is to say that in order to obtain a given emergent brightness, the input energy flux must increase as the auroral width decreases.

\section{A.2. Characteristics of auroral emission produced from Maxwellian electron distributions}

Before applying our model to observations, it is interesting to explore the characteristics of the auroral emission resulting from 2 to $1000 \mathrm{keV}$ single Maxwellian distributions computed in the Grodent et al. (2001) model. All cases used a total energy flux input of $20 \mathrm{~mW} \mathrm{~m}^{-2}$. We first tested the hypothesis that the VER profiles could be approximated by a Chapman profile

$v=v_{m} e^{\left[f\left(1-\alpha-e^{-\alpha}\right)\right]}$,

where $\alpha=\left(z-z_{m}\right) / H$ is the reduced height, $v_{m}$ is the maximum of $v$ at the altitude $z_{m}$ and $f$ is a form factor. Since $f$ and the scale height $H$ influence the shape and FWHM of the VER, $H$ should be considered here as a simple free parameter, not necessary equal to the actual scale height of the emission. We found that the VER originating from these incident Maxwellians could be easily fitted with Chapman profiles. The influence of $\langle E\rangle$ on the emission profiles is summarized in Fig. 12. As seen in Fig. 12a, the altitude of the maximum emission naturally decreases as the mean electron energy increases. The peak altitude slowly decreases and reaches $\sim 160 \mathrm{~km}$ for electrons in a Maxwellian distribution of $\langle E\rangle=500 \mathrm{keV}$. There is very little emission at lower altitudes, which is intuitively explained by the exponential increase of the number of $\mathrm{H}_{2}$ molecules encountered by the electrons with decreasing altitude, which makes it difficult to reach deeper layers, whatever the primary electron energy. The emission peaks at $165 \mathrm{~km}$ for $\langle E\rangle=400 \mathrm{keV}$ and at $163 \mathrm{~km}$ for $\langle E\rangle=500 \mathrm{keV}$. This $2 \mathrm{~km}$ difference leads to a variation of $100 \mathrm{keV}$ in the mean energy determination. The corresponding CR at $\chi=70^{\circ}$ is 20 and 23 for 400 and $500 \mathrm{keV}$, respectively, i.e. a $15 \%$ difference. Since high CR values correspond to strongly
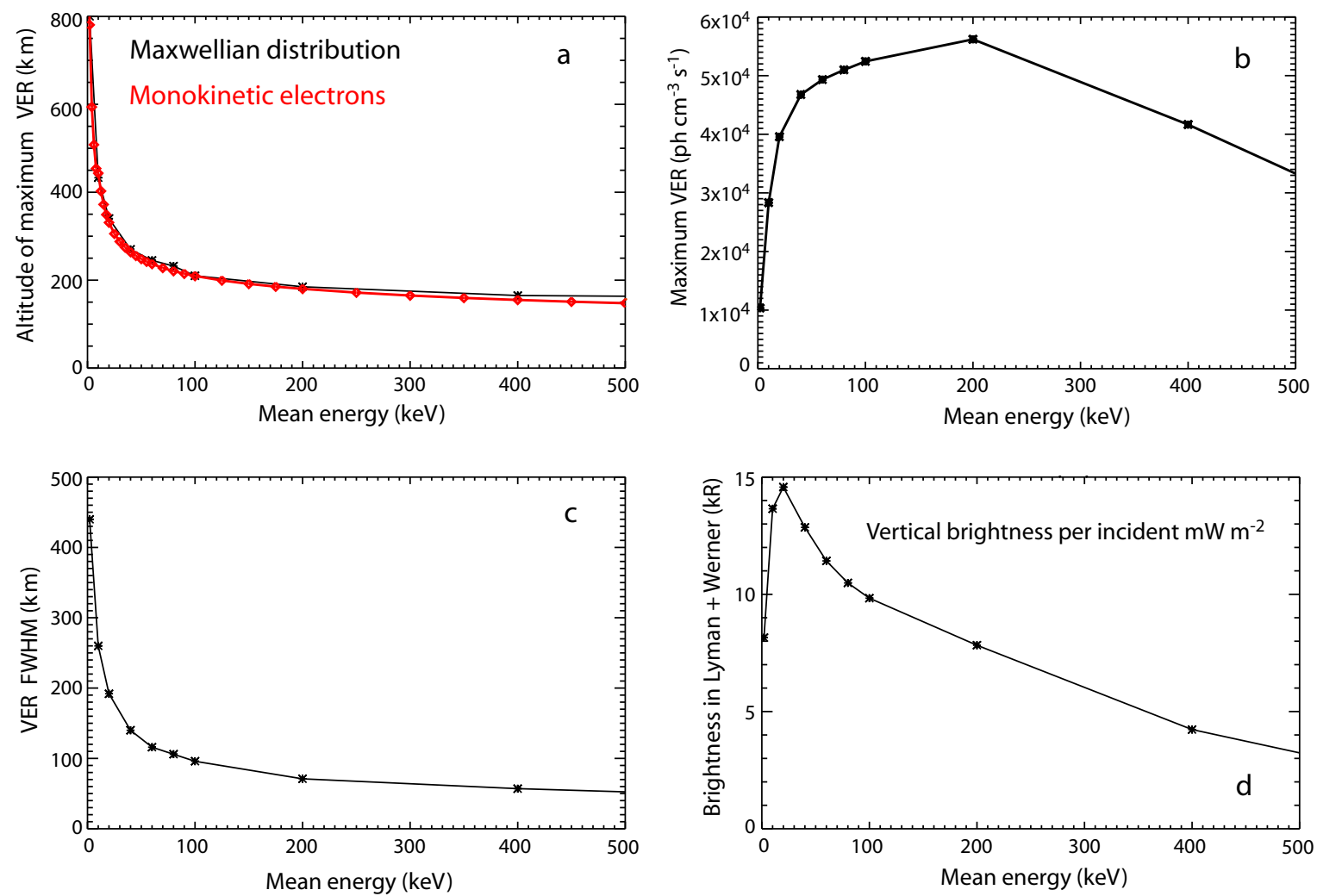

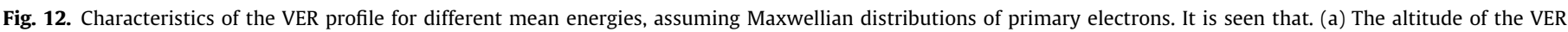

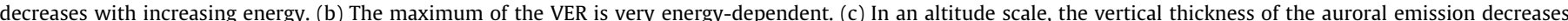

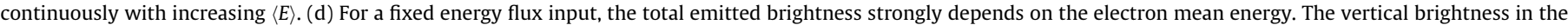
Lyman and Werner bands is maximum at $20 \mathrm{keV}\left(14.6 \mathrm{kR}\right.$ per incident $\left.\mathrm{mW} \mathrm{m}^{-2}\right)$ and then significantly decreases for higher energies. 
attenuated emission in the $1230-1300 \AA$ ange (i.e. emission with low $\mathrm{S} / \mathrm{N}$ ratio), one must be careful in the interpretation of data when high electron energies are involved. Fig. 12a also shows that the altitude of the VER peak resulting from a Maxwellian distribution of electrons at a given $\langle E\rangle$ corresponds very well with the altitudes attained by monokinetic electrons of same energy. The altitude reached by monokinetic electron has been calculated following a continuous slowing down stopping power approximation. The plot in Fig. 12b shows that the maximum value of the VER increases with $\langle E\rangle$, to reach a maximum at $200 \mathrm{keV}$, and then decreases for higher energies. The FWHM of the VER profiles also significantly changes with $\langle E\rangle$ and decreases with increasing energies (Fig. 12c). Although these FWHM remain fixed in pressure scale for different atmospheres, they depend on the pressure-altitude relationship in altitude scale. Values in Fig. 12c are thus only valid in the Grodent et al. (2001) atmosphere, as the temperature profile affects the scale height of the emission. As seen in Fig. 12d for a constant incident energy flux of $1 \mathrm{~mW} \mathrm{~m}^{-2}$, the emergent vertical intensity strongly depends on the mean energy. The efficiency of the atmosphere to create UV auroral emission is maximum for $\langle E\rangle=20 \mathrm{keV}\left(14.6 \mathrm{kR}\right.$ per $\mathrm{mW} \mathrm{m}^{-2}$ ) and then sharply drops for higher energies ( $7.8 \mathrm{kR}$ per $\mathrm{mW} \mathrm{m}^{-2}$ at $200 \mathrm{keV}$ to only $3.2 \mathrm{kR}$ per $\mathrm{mW} \mathrm{m} \mathrm{m}^{-2}$ at $500 \mathrm{keV}$ ). In other words, the energy flux input necessary to create an aurora of given emergent brightness strongly depends on the energy of the precipitating electrons. The value of $10 \mathrm{kR}$ per $\mathrm{mW} \mathrm{m}^{-2}$ based on several aeronomic models and proposed as a rule of thumb by Gustin et al. (2012) should then be reconsidered when the mean electron energy is not in the 70$140 \mathrm{keV}$ range. Since the energies determined in our study exceed this range, values shown in Fig. 12d are used throughout this work. They are particularly important when setting up the energy-energy flux diagrams, as presented in Section 4.2.

\section{References}

Bonfond, B. et al., 2008. UV Io footprint leading spot: A key feature for understanding the UV Io footprint multiplicity? Geophys. Res. Lett. 35, L05107. http://dx.doi.org/10.1029/2007GL032418.

Bonfond, B. et al., 2009. The Io UV footprint: Location, inter-spot distances and tail vertical extent. J. Geophys. Res. 114, A07224. http://dx.doi.org/10.1029/ 2009JA014312.

Bonfond, B. et al., 2011. Quasi-periodic polar flares at Jupiter: A signature of pulsed dayside reconnection? Geophys. Res. Lett. 38, L022104. http://dx.doi.org/ 10.1029/2010GL045981.

Bonfond, B. et al., 2013. Evolution of the Io footprint brightness I: Far-UV observations. Planet. Space Sci. 88, 64-75. http://dx.doi.org/10.1016/j. pps.2013.05.023.

Branduardi-Raymont, G. et al., 2008. Spectral morphology of the X-ray emission from Jupiter's aurorae. J. Geophys. Res. 113 (A2). http://dx.doi.org/10.1029/ 2007JA012600.

Chané, E., Saur, J., Poedts, S., 2013. Modeling Jupiter's magnetosphere: Influence of the internal sources. J. Geophys. Res. 118, 2157-2172. http://dx.doi.org/ 10.1002 /jgra.50258.

Clarke, J.T. et al., 1996. Far-ultraviolet imaging of Jupiter's aurora and the Io "footprint". Science 274, 404. http://dx.doi.org/10.1126/science.274.5286.404.

Clarke, J.T. et al., 1998. Hubble Space Telescope imaging of Jupiter's UV aurora during the Galileo orbiter mission. J. Geophys. Res. 103 (E9), 20217-20236. http://dx.doi.org/10.1029/98JE01130.

Clarke, J.T. et al., 2002. Ultraviolet emissions from the magnetic footprints of Io, Ganymede and Europa on Jupiter. Nature 415 (6875), 997-1000.

Cowley, S.W.H., 2006. Current-voltage and kinetic energy flux relations for relativistic field-aligned acceleration of auroral electrons. Ann. Geophys. 24, 325-338. http://dx.doi.org/10.5194/angeo-24-325-2006.

Cowley, S.W.H., Bunce, E.J., 2001. Origin of the main auroral oval in Jupiter's coupled magnetosphere-ionosphere system. Planet. Space Sci. 49, 1067. http://dx.doi. org/10.1016/S0032-0633(00)00167-7.

Cowley, S.W.H., Bunce, E.J., 2003. Modulation of Jupiter's main auroral oval emissions by solar wind induced expansions and compressions of the magnetosphere. Planet. Space Sci. 51, 57-59. http://dx.doi.org/10.1016/S00320633(02)00118-6.

Dols, V. et al., 2000. Diagnostics of the jovian aurora deduced from ultraviolet spectroscopy: Model and HST/GHRS observation. Icarus 147, 251-266. http:// dx.doi.org/10.1006/icar.2000.6415.
Dumont, M. et al., 2014. Jupiter's equatorial features: Possible signatures of magnetospheric injections. J. Geophys. Res. 119. http://dx.doi.org/10.1002/ 2014JA020527.

Dzifčáková, E., 1998. The ionization equilibrium in the solar corona for the electron power distribution. Sol. Phys. 178, 317-339. http://dx.doi.org/10.1023/ A: 1005087731100

Elsner, R.F. et al., 2005. Simultaneous Chandra X-ray, Hubble Space Telescope ultraviolet, and Ulysses radio observations of Jupiter's aurora. J. Geophys. Res. 110, A01207. http://dx.doi.org/10.1029/2004JA010717.

Gérard, J.-C. et al., 2002. The excitation of the FUV Io tail on Jupiter: Characterization of the electron precipitation. J. Geophys. Res. 107 (A11), 1394. http://dx.doi.org/ 10.1029/2002JA009410.

Gérard, J.-C. et al., 2003. Spectral observations of transient features in the FUV jovian polar aurora. J. Geophys. Res. 108 (A8), 1319. http://dx.doi.org/10.1029/ 2003JA009901.

Gérard, J.C. et al., 2014. Mapping the electron energy in Jupiter's aurora: Hubble spectral observations. J. Geophys. Res. 119 (11). http://dx.doi.org/10.1002/ 2014JA020514.

Gladstone, G.R., Skinner, T.F., 1989. Spectral analysis of jovian auroral emissions. In: Belton, M.J.S., West, R.A., Rahe, J. (Eds.), Proceedings of the Workshop on TimeVariable Phenomena in the Jovian System. NASA SP-494, pp. 221-228.

Gladstone, G.R., Allen, M., Yung, Y.L., 1996. Hydrocarbon photochemistry in the upper atmosphere of Jupiter. Icarus 119, 1-52.

Grodent, D., Waite Jr., J.H., Gérard, J.C., 2001. A self-consistent model of the jovian auroral thermal structure. J. Geophys. Res. 106, 12933-12952. http://dx.doi.org/ 10.1029/2000JA900129.

Grodent, D. et al., 2006. Europa's FUV auroral tail on Jupiter. Geophys. Res. Lett. 33, L06201. http://dx.doi.org/10.1029/2005GL025487.

Grodent, D. et al., 2008. Auroral evidence of a localized magnetic anomaly in Jupiter's northern hemisphere. J. Geophys. Res. 113, A09201. http://dx.doi.org/ 10.1029/2008JA013185.

Gustin, J. et al., 2002. Spatially resolved far ultraviolet spectroscopy of the jovian aurora. Icarus 157, 91-103.

Gustin, J. et al., 2004a. Energy-flux relationship in the FUV jovian aurora deduced from HST-STIS spectral observations. J. Geophys. Res. 109, A10205. http://dx doi.org/10.1029/2003JA010365.

Gustin, J. et al., 2004b. Jovian auroral spectroscopy with FUSE: Analysis of selfabsorption and implications for electron precipitation. Icarus 171, 336-355.

Gustin, J. et al., 2006. Characteristics of jovian morning bright FUV aurora from Hubble Space Telescope/Space Telescope Imaging Spectrograph imaging and spectral observations. J. Geophys. Res. 111, A09220. http://dx.doi.org/10.1029/ 2006JA011730, $13 \mathrm{pp}$.

Gustin, J. et al., 2012. Conversion from HST ACS and STIS auroral counts into brightness, precipitated power, and radiated power for $\mathrm{H}_{2}$ giant planets. J. Geophys. Res. 117, A07316. http://dx.doi.org/10.1029/2012JA017607.

Gustin, J. et al., 2013a. Mapping of the jovian auroral electron energy with HST/STIS observations. European Planetary Science Congress 2013, EPSC Abstracts, vol. 8, EPSC2013-596.

Gustin, J. et al., 2013b. Effects of methane on giant planet's UV emissions and implications for the auroral characteristics. J. Mol. Spectrosc. 291, 108-117. http://dx.doi.org/10.1016/j.jms.2013.03.010.

Gustin, J. et al., 2014. Mapping of the jovian auroral electron energy with HST/STIS observations. American Geophysical Union (Fall). Abstract \#P23A-3978.

Hess, S.L.G. et al., 2010. Power transmission and particle acceleration along the Io flux tube. J. Geophys. Res. 115 (A6). http://dx.doi.org/10.1029/2009JA014928.

Knight, S., 1973. Parallel electric fields. Planet. Space Sci. 21, 741. http://dx.doi.org/ 10.1016/0032-0633(73)90093-7.

Lundin, R., Sandahl, I., 1978. Some characteristics of the parallel electric field acceleration of electrons over discrete auroral arcs as observed from two rocket flights. In: ESA European Sounding Rocket, Balloon and Related Res., pp. 125136.

Maurellis, A.N., Cravens, T.E., 2001. Ionospheric effects of Comet Shoemaker-Levy 9 impacts with Jupiter. Icarus 154, 350-371. http://dx.doi.org/10.1006/ icar.2001.6709.

Moses, J.I. et al., 2005. Photochemistry and diffusion in Jupiter's stratosphere: Constraints from ISO observations and comparisons with other giant planets. J. Geophys. Res. 110 (E8). http://dx.doi.org/10.1029/2005JE002411.

Nichols, J.D., Cowley, S.H.W., 2005. Magnetosphere-ionosphere coupling currents in Jupiter's middle magnetosphere: Effect of magnetosphere-ionosphere decoupling by field-aligned auroral voltages. Ann. Geophys. 23, 799-808. http://dx.doi.org/10.5194/angeo-23-799-2005.

Ozak, N. et al., 2010. Auroral X-ray emission at Jupiter: Depth effects. J. Geophys. Res.: Space Phys. 115 (A11). http://dx.doi.org/10.1029/2010JA015635.

Ozak, N., Cravens, T.E., Schultz, D.R., 2013. Auroral ion precipitation at Jupiter: Predictions for Juno. Geophys. Res. Lett. 40, 4144-4148. http://dx.doi.org/ $10.1002 /$ grl.50812.

Palmaerts, B. et al., 2014. Transient small-scale structure in the main auroral emission at Jupiter. J. Geophys. Res. 119, 9931-9938. http://dx.doi.org/10.1002 2014JA020688.

Parkinson, C.D., Stewart, A.I.F., Wong, A.S., Yung, Y.L., Ajello, J.M., 2006. Enhanced transport in the polar mesosphere of Jupiter: Evidence from Cassini UVIS helium 584 Å airglow. J. Geophys. Res. 111, E02002. http://dx.doi.org/10.1029/ 2005JE002539.

Prangé, R. et al., 1996. Rapid energy dissipation and variability of the Io-Jupiter electrodynamic circuit. Nature 379, 323-325. http://dx.doi.org/10.1038 379323a0. 
Prangé, R. et al., 1998. Detailed study of FUV jovian auroral features with the postCOSTAR HST faint object camera. J. Geophys. Res. 103, 20195-20216. http://dx doi.org/10.1029/98JE01128.

Radioti, A. et al., 2008. Discontinuity in Jupiter's main auroral oval. J. Geophys. Res. 113 (A1). http://dx.doi.org/10.1029/2007JA012610.

Radioti, A. et al., 2009. Equatorward diffuse emissions at Jupiter: Simultaneous HST and Galileo observations. Geophys. Res. Lett. 36. http://dx.doi.org/10.1029/ 2009GL037857.

Ray, L.C. et al., 2009. Current-voltage relation of a centrifugally confined plasma. J. Geophys. Res. 114, A04214. http://dx.doi.org/10.1029/2008JA013969.

Ray, L.C. et al., 2010. Magnetosphere-ionosphere coupling at Jupiter: Effect of fieldaligned potentials on angular momentum transport. J. Geophys. Res. 115 (A9). http://dx.doi.org/10.1029/2010JA015423.

Sada, P.V., Bjoraker, G.L., Jennings, D.E., McCabe, G.H., Romani, P.N., 1998 Observations of $\mathrm{CH}_{4}, \mathrm{C}_{2} \mathrm{H}_{6}$ and $\mathrm{C}_{2} \mathrm{H}_{2}$ in the stratosphere of Jupiter. Icarus 136 (1998), 192. http://dx.doi.org/10.1006/icar.1998.6021.

Scudder, J.D., Sittler Jr., E.C., Bridge, H.S., 1981. A survey of the plasma electron environment of Jupiter: A view from Voyager. J. Geophys. Res. 86, 8157. http:/ dx.doi.org/10.1029/JA086iA10p08157.
Seiff, A. et al., 1996. Structure of the atmosphere of Jupiter: Galileo probe measurements. Science 272, 844-845. http://dx.doi.org/10.1126/science.272. 5263.844 .

Smith, F.L., Smith, C., 1972. Numerical evaluation of Chapman's grazing incidence integral $\mathrm{Ch}(X, \chi)$. J. Geophys. Res. 77, 3592-3597. http://dx.doi.org/10.1029/ JA077i019p03592.

$\mathrm{Su}$, Y.-J. et al., 2003. Io-related jovian auroral arcs: Modeling parallel electric fields. J. Geophys. Res. 108 (A2), 1094. http://dx.doi.org/10.1029/2002JA009247.

Waite Jr., J.H. et al., 2001. An auroral flare at Jupiter. Nature 410, 787-789.

Wannawichian, S., Clarke, J.T., Nichols, J.D., 2010. Ten years of Hubble Space Telescope observations of the variation of the jovian satellites' auroral footprint brightness. J. Geophys. Res. 115 (A2). http://dx.doi.org/10.1029/2009JA014456.

Yung, Y.L. et al., 1982. $\mathrm{H}_{2}$ fluorescence spectrum from 1200 to $1700 \AA$ by electron impact: Laboratory study and application to jovian aurora. Astrophys. J. 254, L65-L69. http://dx.doi.org/10.1086/183757. 